Check for updates

Cite this: RSC Adv., 2018, 8, 34012

Received 19th July 2018

Accepted 27th September 2018

DOI: $10.1039 / \mathrm{c} 8 \mathrm{ra06132g}$

rsc.li/rsc-advances

\section{The potential of paper-based diagnostics to meet the ASSURED criteria}

\author{
Suzanne Smith, (D) *a Jan G. Korvink, (D) ${ }^{\mathrm{b}}$ Dario Mager ${ }^{\mathrm{b}}$ and Kevin Land (D) ${ }^{\mathrm{a}}$
}

Paper-based diagnostics have already revolutionized point-of-care approaches for health and environmental applications, by providing low-cost, disposable tools that can be utilized in remote settings. These devices typically consist of microfluidic, chemical, and biological diagnostic components implemented on paper substrates, towards addressing the ASSURED (Affordable, Sensitive, Specific, User friendly, Rapid and Robust, Equipment free and Deliverable to end users) principles set out by the World Health Organization. Paper-based diagnostics primarily contribute to the affordable, equipment-free, and deliverable-to-end-user aspects. However, additional functionality must be integrated with paperbased diagnostic devices to achieve truly ASSURED solutions. Advances in printed electronics provide a fitting foundation for implementing augmented functionality, while maintaining the affordability and disposability of paper-based diagnostics. This paper reviews the printed functional building blocks that contribute towards achieving this goal, from individual printed electronic components to fully integrated solutions. Important modules for sensing, read-out of results, data processing and communication, and on-board power are explored, and solutions printed on flexible or paper-based substrates for integration with paper-based diagnostics are considered. Although many of the unit operations required to achieve the ASSURED criteria can be implemented using paper, basic system functionality is still lacking, and this requires a concerted effort in integration of the various components for truly ASSURED solutions to be realized. Beyond ASSURED, modern clinical practises and crisis readiness also require additional informational functionality, which a systems approach using paper-based solutions could ensure.
${ }^{a}$ Council for Scientific and Industrial Reasearch (CSIR), Pretoria, South Africa. E-mail: ssmith@csir.co.za; Tel: +27 128413101
${ }^{b}$ Karlsruhe Institute of Technology (KIT), Karlsruhe, Germany

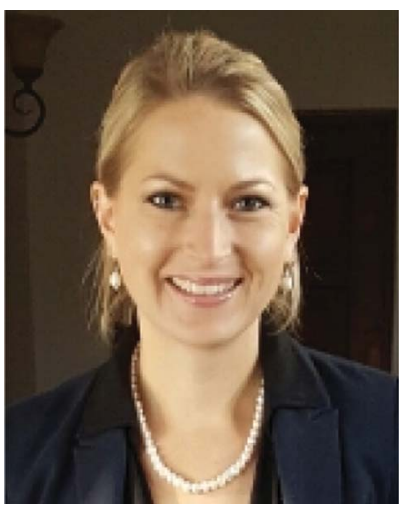

Suzanne Smith obtained her B Eng Electronic Engineering degree in 2006, and her M Eng Bioengineering degree (specializing in music perception in cochlear implantees) in 2010, both from the University of Pretoria, South Africa. She is currently registered for a PhD in Microsystems at the Karlsruhe Institute of Technology (KIT), Germany, while working in the Microsystems group at the Council for Scientific and Industrial Research (CSIR), South Africa. Her area of research interest is in the field of microsystems and microfluidics, with focus on printed electronics for low-cost pointof-care health and environmental diagnostics.

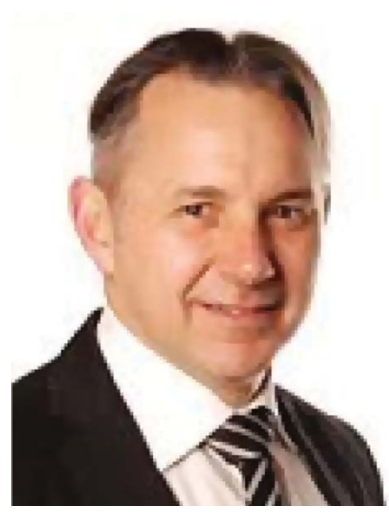

Jan G. Korvink received his M.Sc. degree in mechanical engineering (specializing in computational mechanics) from the University of Cape Town, South Africa, in 1987, and his Ph.D. degree from the ETH Zurich, Switzerland, in 1993. After this, he joined the Physical Electronics Laboratory, ETH Zurich. In 1997, he joined the University of Freiburg, Freiburg, Germany, as full professor for microsystems engineering. In Freiburg he co-directed the Freiburg Institute for Advanced Studies (FRIAS). In 2015 he joined KIT, where he directs the Institute of Microstructure Technology (IMT). His research interests include the development of ultralow-cost micromanufacturing methods, microsystem applications in the area of magnetic resonance imaging, and the design and simulation of micro- and nanosystems. 


\section{Introduction}

In recent years, there has been a drive to develop low-cost, innovative point-of-care diagnostics to reach populations where the burden of disease is greatest, and where resources are fewest. These developments have been guided by the World Health Organization (WHO), through formalization of the ASSURED criteria - Affordable, Sensitive, Specific, User friendly, Rapid and Robust, Equipment-free and Deliverable to end users - to which these diagnostics should conform if they are to be successful in resource-limited settings. ${ }^{1}$ Here, we review we explore various printed functional components that are currently available or under development towards achieving diagnostics that fulfill these criteria.

Paper-based microfluidics provide a favourable platform on which to develop solutions that address these requirements, ${ }^{2,3}$ with paper exhibiting a number of useful properties that are either inherent or that can be achieved through straightforward modifications. This includes the low cost, recyclability and disposability of paper to provide devices that can easily be transported, distributed, and safely incinerated. Paper enables automated fluidic handling through a porous substrate, with simple, visual result readout by the user typically implemented via a colour change. Paper-based solutions also facilitate high quality mass manufacturing which is based on a long tradition of paper processing.

Printed functionality can be incorporated with these devices, stemming from printed materials development and printed electronics fields ${ }^{4-7}$ to result in devices with enhanced userfriendliness through automated result read-out and communication. This also removes the need for training or external instrumentation to correctly perform a test. Building blocks from the field of printed electronics, such as sensors, processors, displays, connectivity and power have the potential to be incorporated to extend the functionality of these devices.
Paper-based solutions have successfully been applied to health, environmental and food quality monitoring. ${ }^{8,9}$ Colorimetric, electrochemical, or fluorescence based analysis methods ${ }^{\mathbf{1 0}}$ are typically used, relying on either external equipment for analysis, or on visual colorimetric read-out, with the latter being prone to errors and typically not providing quantitative results. More sophisticated result read-out generally requires some form of external instrumentation to perform processing and communication of the result from the paperbased test ${ }^{\mathbf{1 1}}$ (Fig. 1). Common methods include the use of existing devices such as scanners, portable glucose meters and mobile phones, ${ }^{12,13}$ as well as custom-developed systems such as portable potentiostats, ${ }^{\mathbf{1 4 - 1 6}}$ many of which have been extensively reviewed. $^{2}$

Limited network and maintenance infrastructure, intermittent power, and issues with reliable storage in resource-limited settings create challenges in the successful implementation of instrumented solutions. This highlights the need for quantitative detection, read-out and connectivity to be integrated in to an automated, maintenance-free system, ideally incorporated on the diagnostic device itself. For such a solution, the result would need to be expressed to the user, possibly through a visual display on the device.

Capturing, conveying and storing of the test results would also be required, as this is a significant challenge in point-ofcare testing, particularly in South African clinics. ${ }^{17,18}$ Visualbased tests such as standard lateral flow test strips are subject to user bias, with incorrect result readout a common occurrence. ${ }^{19}$ Additionally, these manual tests do not allow for effective communication of the result or diagnosis, or for data collection for assessment of epidemics or therapy strategies. Automated result readout and communication would enable this data to be captured and analysed, contributing to various health system needs. To facilitate the effective implementation of read-out, displays and communication modules, on-board

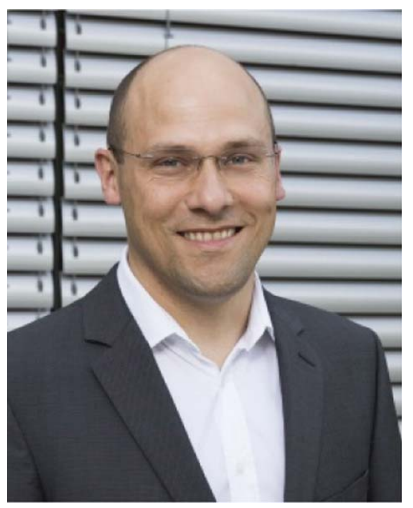

Dr Dario Mager leads the lowcost MEMS group at IMT at KIT in Germany. For his work on the fabrication of microstructures using inkjet printing he obtained his PhD in MEMS at the University of Freiburg (IMTEK), Germany. He and his team work on MEMS devices that can be fabricated without a cleanroom. The naturally lower level of quality is compensated by using embedded systems for better signal readout and analysis. In this context, the most complex system is the eLoaD platform where centrifugal microfluidics are enhanced through the co-integration of embedded systems in the spinning frame of reference.

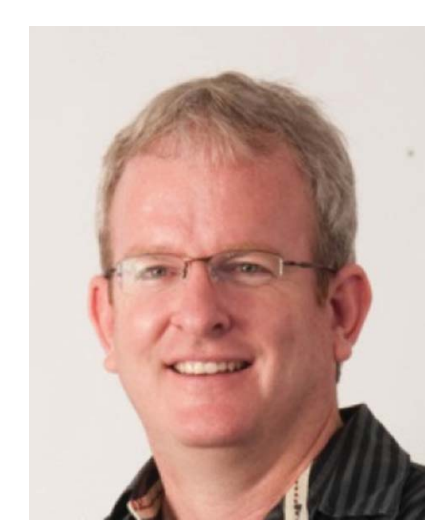

Dr Kevin Land is the platform leader of the microsystems technology group at the CSIR in South Africa. Kevin established the microsystems and microfluidics technology capabilities at CSIR. Dr Kevin Land obtained a PhD in Microsystems Engineering through the Institute for Microsystems Technology (Imtek), University of Freiburg, Germany, where the main focus was on the development of microfluidic droplet systems. He has a passion to develop equipment free or minimally instrumented low cost diagnostics solutions utilising printed functionality, low cost substrates and microfluidics - particularly for developing countries where such solutions would have massively transformative impact and have the opportunity to reach billions of people. 


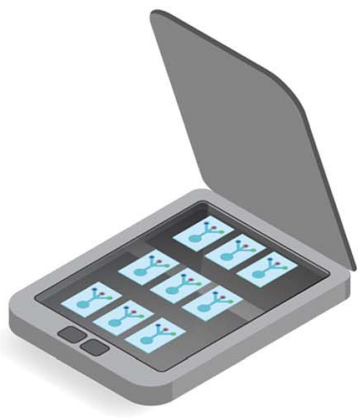

A

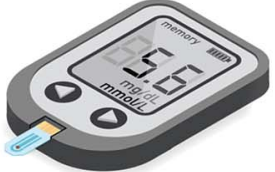

B

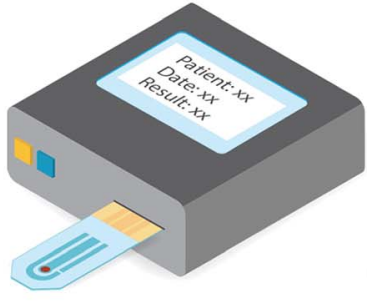

C

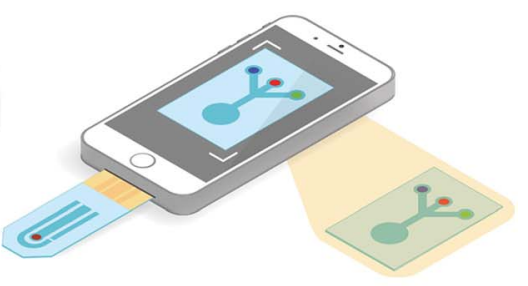

D

Fig. 1 Examples of existing external instrumentation solutions that can be used with paper-based diagnostic tests. (A) a desktop scanner, (B) a portable glucose meter used with custom made paper-based diagnostic electrochemical sensors, (C) a custom-made instrument for analysis of paper-based diagnostic test devices, and (D) a phone camera for image capture and processing of a paper-based device and/or for physical connection and analysis of a paper-based device.

power may be required to drive these added functionalities, without relying on external power sources.

Taking into account these various challenges and unmet needs, a fully ASSURED device would require the following functional components, each contributing to improvements in different ASSURED aspects:

- Actuation and control modules for operation of and user interaction with the device, improving usability and providing built-in instrumentation,

- Microfluidics for processing and fluidic control of the sample to be tested, contributing to low-cost diagnostics with high specificity,

- Electronics to add sensitivity and speed towards automated, integrated testing and instrumentation,

- Sensing modules towards built-in, fast and sensitive detection techniques and instrumentation,

- Data processing capabilities to analyse the sample accurately and automatically and capture the result digitally, contributing to most aspects of ASSURED,

- Read out and display modules to express the result directly to the user, improving result read-out times and userfriendliness with built-in instrumentation,

- Connectivity for transmission and storage of results to be accessed remotely or in future as needed, contributing to many ASSURED aspects, and

- Energy storage for built-in power to drive the various functional components, assisting with deployment of devices with on-board instrumentation.

This work discusses each of these aspects and their integration towards the practical implementation of fully ASSURED devices. Fig. 2 shows a conceptual overview of the various functional modules combined in to an automated, integrated paper-based diagnostic solution, along with a graphical outline of the review with reference to the functional modules defined. The envisaged device utilizes a small sample of plasma or blood, processes the sample, reads out the result through sensing and processing steps, and obtains a digitized result that can be transmitted and stored, thus directly diagnosing a targeted disease.

\section{Microfluidics and sample processing}

Paper tests such as $\mathrm{pH}$ strips and home-based lateral flow pregnancy tests are examples of the first and most common paper-based microfluidic devices ${ }^{3}$ (Fig. 3A). Enhanced functionality such as multiplexing and multi-dimensional fluidic handling can be garnered from advances in paper-based microfluidics, also referred to as microfluidic paper-based analytical devices ( $\mu$ PADs) ${ }^{20}$ through multi-channel designs within a paper substrate (Fig. 3B), or by stacking and folding of paper in to so-called three-dimensional or "origami" paperbased microfluidics ${ }^{10}$ (Fig. 3C and D). Wax printing of fluidic channels is often implemented using hydrophobic barriers to contain and guide fluids through a paper device. Inkjet printing, screen printing and flexographic printing have also been utilized. ${ }^{21,22}$ Modifications in the composition of the paper substrate allow for heightened functionality of paper-based diagnostics ${ }^{23}$ and printing of paper substrates themselves are currently being explored, for example by using printable nanocellulose pulp. The use of nanocellulose for implementing printed electronics has gained attention ${ }^{6}$ and along with advances in paper-based microfluidics and diagnostics, ${ }^{2,22}$ forms the foundation on which to further develop augmented printed functionality for paper-based diagnostics.

Paper-based microfluidics allow for functions such as sample filtering, fluidic movement and splitting of fluids in to different reaction zones for multiplexed testing to be achieved, and have been implemented for various biological samples including whole blood, serum, urine and nasal swabs. ${ }^{2}$ These paper-based microfluidics have been integrated with a variety of sensors for electrochemical, colorimetric or other detection techniques ${ }^{3}$ to achieve automated sample processing and detection components towards the envisaged ASSURED device shown in Fig. 2.

\section{Electronics}

Augmented functionality for paper-based diagnostics will require a number of individual printed passive and active 


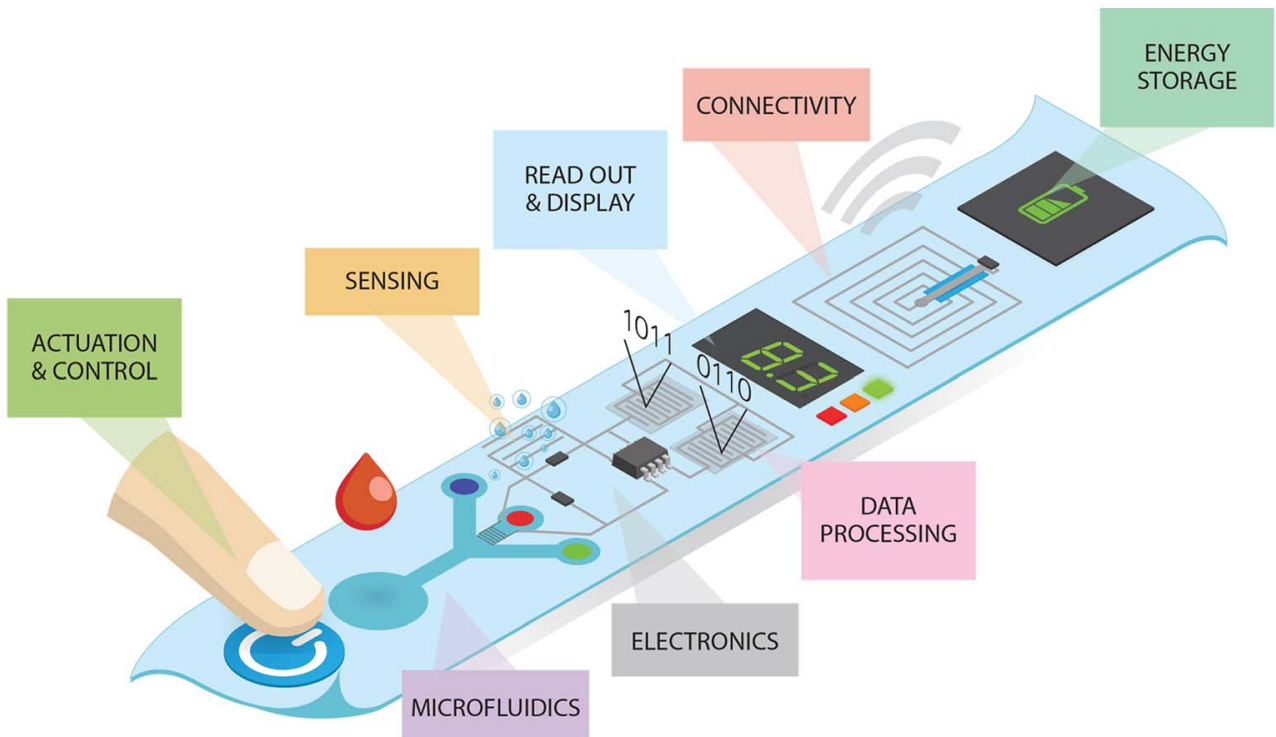

A

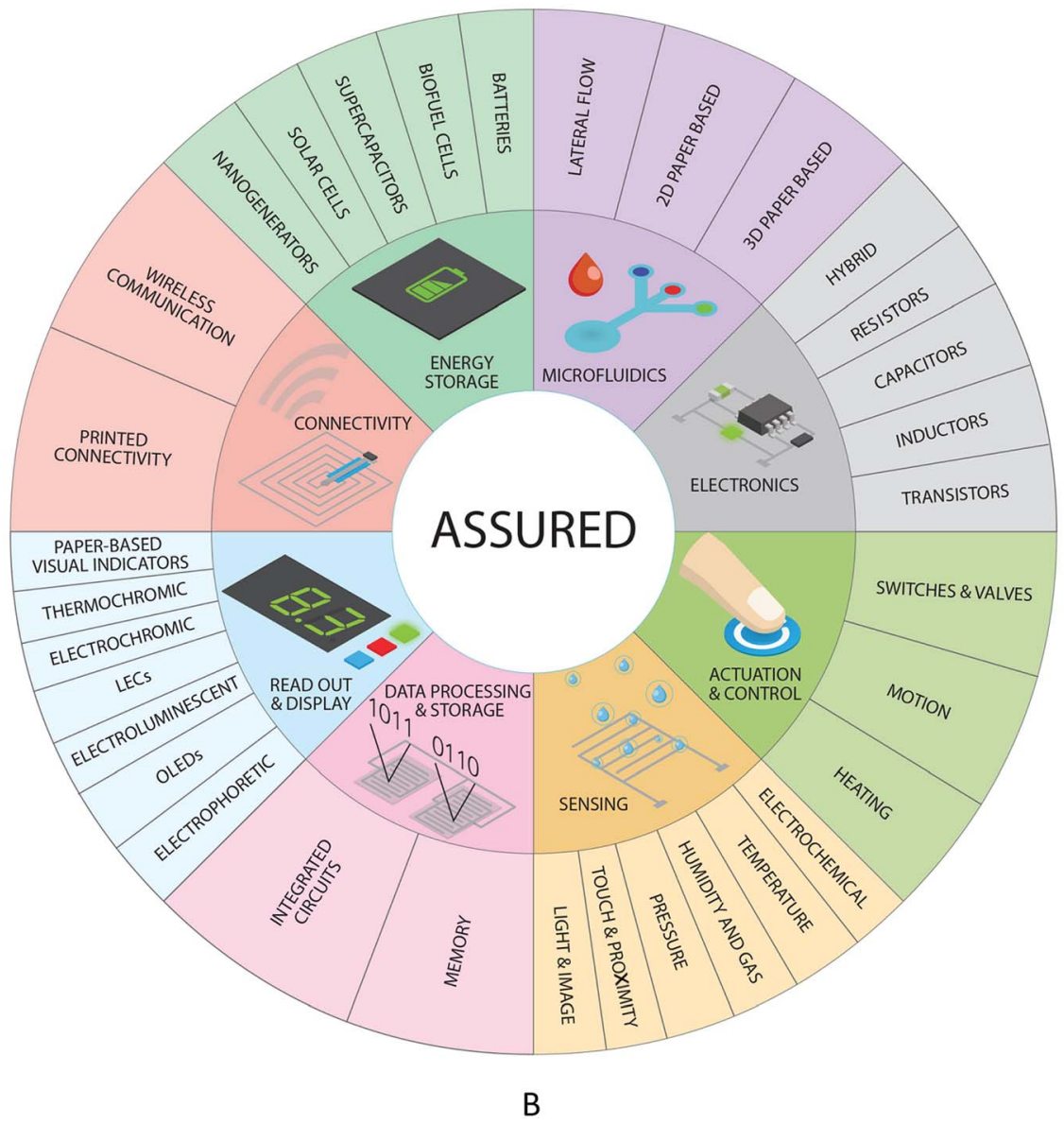

Fig. 2 (A) Envisaged integrated ASSURED device with various functional modules indicated, and (B) graphical summary of the review highlighting the printed functionalities that can be utilized to realize integrated, ASSURED devices.

electronic components to build up more complex electronic circuitry and enable processing capabilities for read-out of results. Advances in printed electronics ${ }^{24,25}$ provide both hybrid and fully printed approaches (Fig. 4) on flexible, paper-based substrates using printing methods such as screen, inkjet, gravure, flexographic, off-set, slot-die, transfer printing, as well as micro-contact and nanoimprinting. ${ }^{5,26}$

Parallels can be drawn between printed electronics and integrated circuit (IC) manufacturing technologies, where in the 1960s, achievable IC lithography line widths were in the region 


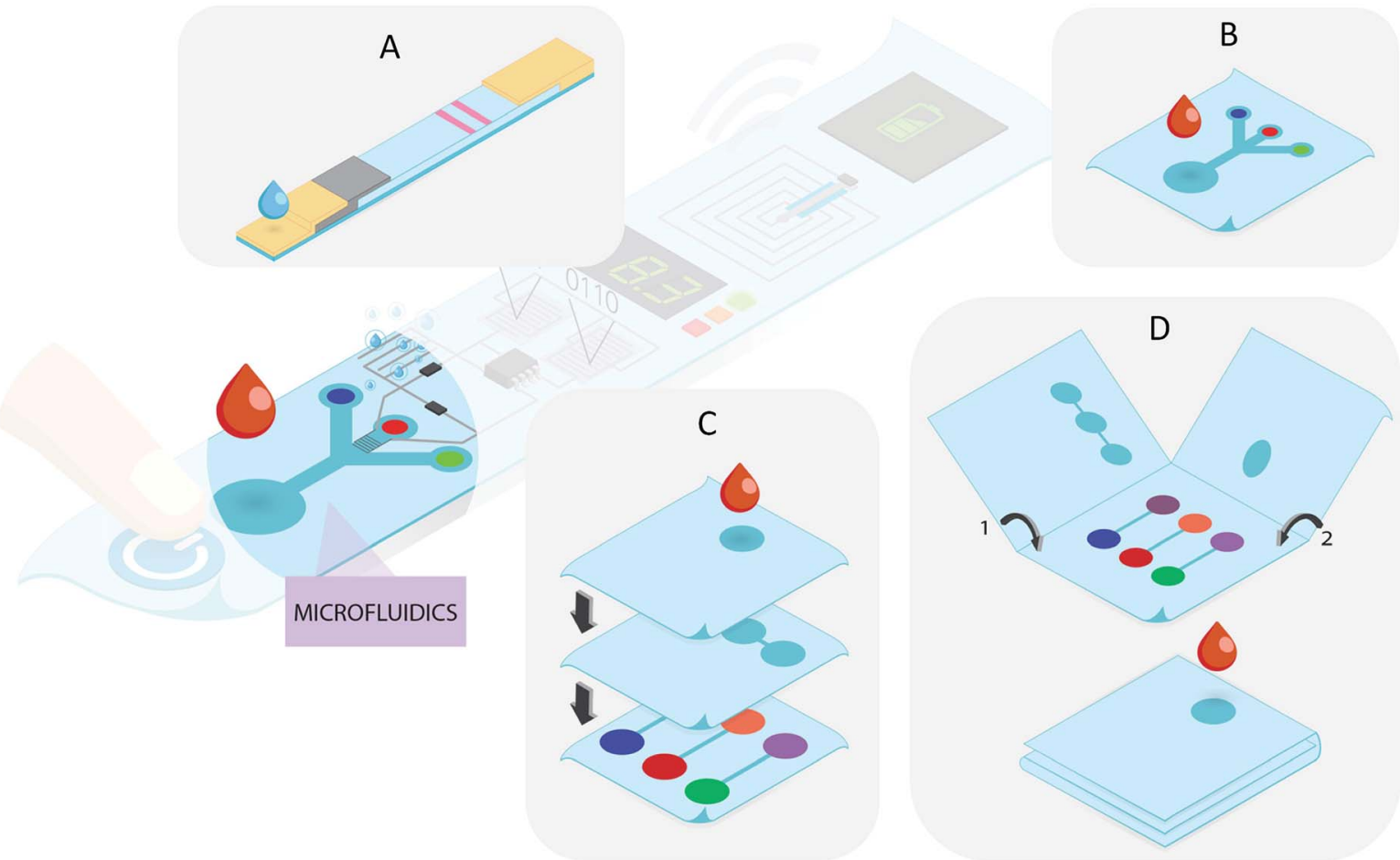

Fig. 3 Microfluidics and sample processing functional modules, illustrating typical paper-based diagnostic test formats. (A) Standard lateral flow test strips with sample pad, conjugate pad, test and control lines and wicking pad, (B) two-dimensional paper-based diagnostics using hydrophobic fluidic barriers (e.g. wax printing), and three-dimensional paper-based diagnostics implemented using (C) stacking of multiple layers or (D) folding or origami paper devices for enhanced fluidic control and multiplexing.

of $10 \mu \mathrm{m}$. This is similar to the resolution limit currently achievable using printing technologies such as inkjet printing for electronics, ${ }^{27}$ highlighting the significant progress still required by printed and paper-based electronics. Hybrid printed electronics combine printed, flexible electronics with existing IC technologies, with successful integration of bare dies with printed electronics a possibility, improving the flexibility and lowering the cost of the circuit. Advances in resolution limits for printed electronics continue to be made, and fully printable components realized, including gravure printed transistors with channel widths as small as $5 \mu \mathrm{m} .{ }^{28}$

Conductive silver, copper or carbon-based inks are typically used to print electronic tracks, electrodes and antennas. Transparent conductors such as indium tin oxide (ITO) and more recently poly(3,4-ethylenedioxythiophene) polystyrene sulfonate (PEDOT:PSS) and graphene are utilized in printing of components such as solar cells and organic light-emitting diodes (OLEDs). ${ }^{27}$ Once printed, UV or low-temperature curing is used to remove the solvent and form solid, conductive features that adhere to the substrate. Additional printed materials include semi-conductors and dielectrics. ${ }^{5,26}$ Both inorganic and organic materials can be printed, and various commercially available functional inks enable printed functionality to be implemented. Control of geometrical dimensions is important for realizing functional printed electronics, and thus a balance between the printed functionality and the substrate suitability to paper-based diagnostics can prove challenging and continues to be explored. ${ }^{5,29}$

\subsection{Hybrid printed electronics}

A hybrid approach involves utilizing existing surface mount device (SMD) packaged electronic components - from resistors to light-emitting diodes (LEDs) and ICs - that can be mounted on to printed tracks on flexible paper substrates ${ }^{30-32}$ (Fig. 4A). Low temperature soldering, conductive epoxies and crimping can be used to secure and connect the components to the printed tracks, and compatibility of these with the paper substrate is an important consideration. The rigidity of the packaged components can also limit the flexibility of the device, but bare die formats or small, thin and flexible packaging can alleviate these challenges to a certain extent. Integration using pick and place equipment for the components is feasible, ${ }^{27}$ but in smaller quantities, remains a manual process.

\subsection{Printed electronic components}

Printed electronic components have been realized on various flexible and paper-based substrates. ${ }^{33-35}$ Temperature, bending, and moisture, among other factors, can affect the performance and repeatability of these components. ${ }^{27}$

3.2.1 Resistors. Resistors can be printed simply by varying the resistivity and geometrical dimensions (length, cross sectional area) of a conductive ink - typically carbon - that is printed over the ends of two metallic conductive track terminals (Fig. 4B). Dimensions for widths and lengths are typically in the $\mathrm{mm}$ range with heights in the $\mu \mathrm{m}$ range, depending on the printing technique chosen. ${ }^{27}$ The simple design and 


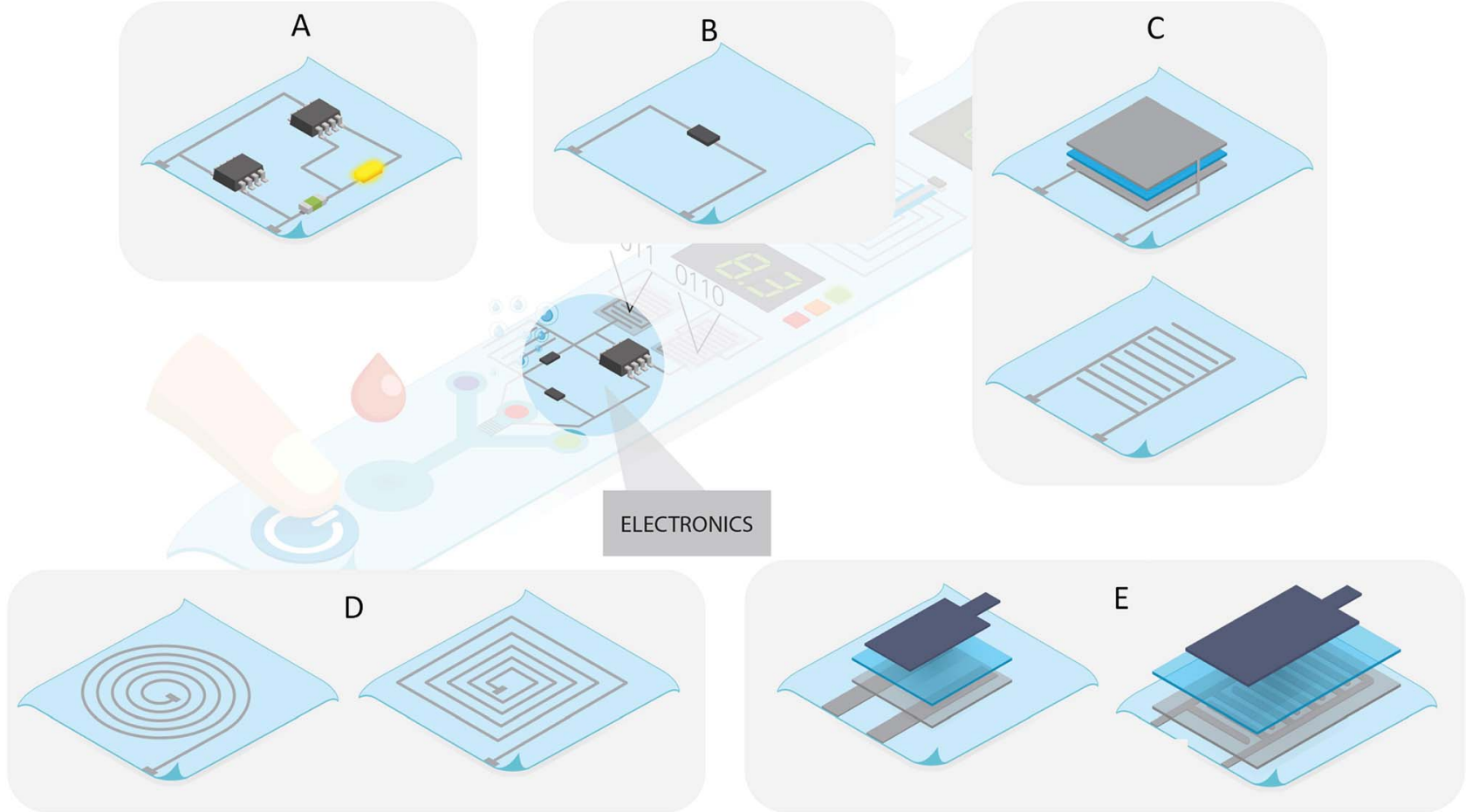

Fig. 4 Printed paper-based electronics. (A) Hybrid printed electronics with surface mount components such as ICs and LEDs, mounted on to printed circuit tracks on paper. (B) Printed resistor with silver tracks and a resistive material to connect the terminals and create a resistive element. (C) Printed capacitor structures, showing both stacked and interdigitated formats. A dielectric material is printed between two conductive electrodes in the stacked format. (D) Printed inductor design with conductive tracks printed in circular or square spiral shapes. (E) Printed transistor with various functional layers labelled (left) and a typical printed transistor layout (right).

manufacture process enables various resistor values to be realized as fully printable, accurate and repeatable resistive components that can easily be integrated in to existing printed electronic solutions. ${ }^{34,36}$

3.2.2 Capacitors. Interdigitated capacitors can be implemented through lateral printed structures consisting of fingerlike or comb designs. Capacitances achievable with these designs are limited to the picofarad $(\mathrm{pF})$ range. In cases where greater capacitances are required, stacked structures can be utilized to achieve capacitance values in the nanofarad ( $\mathrm{nF}$ ) range. In these designs, a dielectric layer is printed between two conducting electrode layers (Fig. 4C). Thicknesses of at least 5 $\mu \mathrm{m}$ are typically used for the dielectric layers, often printed in multiple layers to prevent short circuits. Typical values for standard printing processes are less than $5 \mathrm{nF}$, but values in the $\mu \mathrm{F}$ range can be achieved in special cases. ${ }^{27}$

3.2.3 Inductors. Inductors can be realized by surrounding an enclosed area, for example, by printing planar square or circular spiral shapes ${ }^{33-35}$ (Fig. 4D). The total inductance is calculated from the self-inductances of the straight segments of the spiral shape, as well as the mutual inductances resulting from the magnetic fields of adjacent branches of the inductor. ${ }^{27}$ The conductivity of the spiral track as well as the width and length of the track segments affect the resistance and thus the quality factor of the inductor, and printing resolution is key. Multilayer printing and alignment is required to provide a connection from the inside of the spiral to the outside of the inductor shape across a good insulator to avoid leakage current. Typical values achievable are in the range of a few $\mu \mathrm{H}$ for inductors operating at $100 \mathrm{kHz}{ }^{27}$

3.2.4 Transistors. Transistors are important in almost all electronic systems as switches or amplifiers. Printed transistors make use of multilayer printing processes and different materials $^{37,38}$ and typically mimic the functionality of a field-effect transistor. They consist of a gate, drain and source, with dielectric and semiconductor layers (Fig. 4E). Paper-based inorganic and organic transistors - the latter with an organic semi-conductor - have been developed. Cellulose-based paper is often used as a substrate or a gate dielectric for paper-based transistors, where the gate electrode can be printed on one side of the paper and the semiconductor and source/drain electrodes on the other. ${ }^{39}$ Some examples include inkjetprinted organic transistors ${ }^{40,41}$ and flexible, transparent transistors on nanopaper, ${ }^{42}$ as well as more recent flexible inorganic/organic hybrid transistors that showcase high performance. ${ }^{43}$ Low-cost arrays of printed transistors on to paper substrates have also been demonstrated. ${ }^{44}$ Printing resolution of transistors remains an important consideration to achieve the desired performance, but gravure printed transistors with channel widths of as low as $5 \mu \mathrm{m}$ have been realized, ${ }^{28}$ showing promise for this area of development.

Electrochemical transistors (ECTs) are gated by an electrolyte and show promise for ASSURED devices as they operate at low potentials, typically around 0.5 to $2 \mathrm{~V}$. ECTs have been 
implemented in disposable alcohol breathalizers, ${ }^{45}$ wearable biosensors ${ }^{\mathbf{4 6}}$ and have been developed with fast switching times by Acreo and Linköping University ${ }^{47}$ for use in logic circuits and active-matrix displays.

Crucial parameters still to be optimized for paper-based transistors include low operation voltages, high stability and high carrier mobility, with nanopaper showing promise as a potential substrate to address these challenges. ${ }^{48}$

Fully printed electronics cannot currently compete with silicon electronic technologies in terms of speed, and thus new technologies for flexible, thin silicon devices are being developed towards a Moore's Law for flexible electronics. ${ }^{49}$ This would map a path for the mass production of more and more densely populated flexible circuits. Initial developments utilize metal oxide semiconductors to create thin film transistors that move charge significantly faster than their organic counterparts, and can be fabricated at room temperature. Integration of these advances with other paper-based functional components would remain a critical challenge in future developments.

While fully printed electronics continue to advance in terms of performance, hybrid solutions with both printed and existing packaged components can already implement many unit operations to build up electronic circuits on paper-based devices. These could be used as building blocks as part of sensors, processors, display and connectivity components in an automated, integrated paper-based ASSURED diagnostic device.

\section{Actuation and control}

User interaction with the integrated paper-based diagnostic device will enable the test to be initiated or operational steps to be performed. Both electronic and fluidic switches and valves can be implemented for paper-based devices. Commercial flexible membrane switches provide a simple solution using printed conductive tracks with spacers that cause the switch to close when the button is pressed (Fig. 5A), while fluidic switches for timing, programming and other functional fluidic control in paper devices have been explored ${ }^{10,20,32,50}$ (Fig. 5B).

\subsection{Motion}

Fluidic movement in paper-based devices is facilitated by the inherent capillary action that paper exhibits and can be enhanced through fluidic switching and flow patterns in paper, as previously mentioned. Motion can also be achieved through physical actuators and piezoelectrics implemented on paper.

Paper-based actuators enable physical movement to be realized on paper devices by undergoing dimensional changes as a result of moisture variations, ${ }^{32} r$ can be implemented using electroosmotic actuators, ${ }^{51}$ often made from cellulose and hybrid materials. ${ }^{52}$ High-performance paper actuators using standard printing paper and based on electrothermal activation have recently been demonstrated ${ }^{53}$ (Fig. 5C). The low voltage, power and temperature requirements of these actuators are thus well-suited for application in low-cost paper-based diagnostics where additional movement or control may be required.
Piezoelectric papers have been developed, with examples made from functionalized wood cellulose fibers ${ }^{54}$ and are typically used for pressure and force sensing applications, ${ }^{55}$ which are discussed in Section 5. Piezoelectric papers with high flexibility and tensile strength have been realized for use as tactile sensors, ${ }^{56}$ similar to membrane switches and push-buttons for actuation. Environmentally friendly piezoelectric paper for sensing and actuation has also been developed, with actuation of paper cantilevers presented as an example application. ${ }^{57}$ This contributes to the development of environmentally friendly micro-electro-mechanical systems (MEMS) with improved foldability and affordability, and which are well-suited to the progression of robust and low-cost point-of-care paper-based diagnostic solutions.

Mechanical actuation using buttons, switches and movement could already be integrated with paper-based diagnostic devices in a straightforward manner for user interaction with the device. Fluidic actuation requires more precise implementation and integration considerations, but is also feasible for assisting with sample introduction and processing.

\subsection{Heating on paper}

Heating is important in various chemical and biological reactions and can facilitate sample concentration on paper through evaporation of liquids, or can support the implementation of valves on paper-based devices. ${ }^{58,59}$ Advances in materials for printed resistors allows for pastes with either positive or negative temperature coefficients to be printed. These inks can be used as self-regulating heating elements, where a specified temperature can be reached and maintained when a current is applied, without the need for additional control and regulating circuitry (Fig. 5D). Carbon nanotubes have also been printed on paper and textiles to implement heating elements. ${ }^{\mathbf{6 0}}$

Paper-based heaters assist in sample preparation and processing, e.g. for implementing controlled heating cycles for polymerase chain reaction (PCR), towards fully ASSURED devices. Paper-based heaters could also be implemented onboard an ASSURED device for effective control of thermochromic displays (Section 7) to display a result to the user. Effective paper-based heater prototypes operating from a $5 \mathrm{~V}$ supply can reach temperatures of over $50{ }^{\circ} \mathrm{C}$. These devices can be implemented directly as part of integrated ASSURED devices, contributing to sample processing and display components.

\section{Sensing}

Integrated sensing or detection techniques for paper-based diagnostics include colorimetric, electrochemical, electrical conductivity and chemiluminescent methods. ${ }^{8}$ Printed sensors on flexible substrates ${ }^{61}$ and printed MEMS $^{62}$ have been comprehensively reviewed. Printed resistive sensors form a large part of the printed sensing capabilities on paper substrates where foldable, low-cost designs ${ }^{36}$ and multisensory platforms for temperature, humidity, pressure, $\mathrm{pH}$ and flow have been implemented. ${ }^{63}$ 


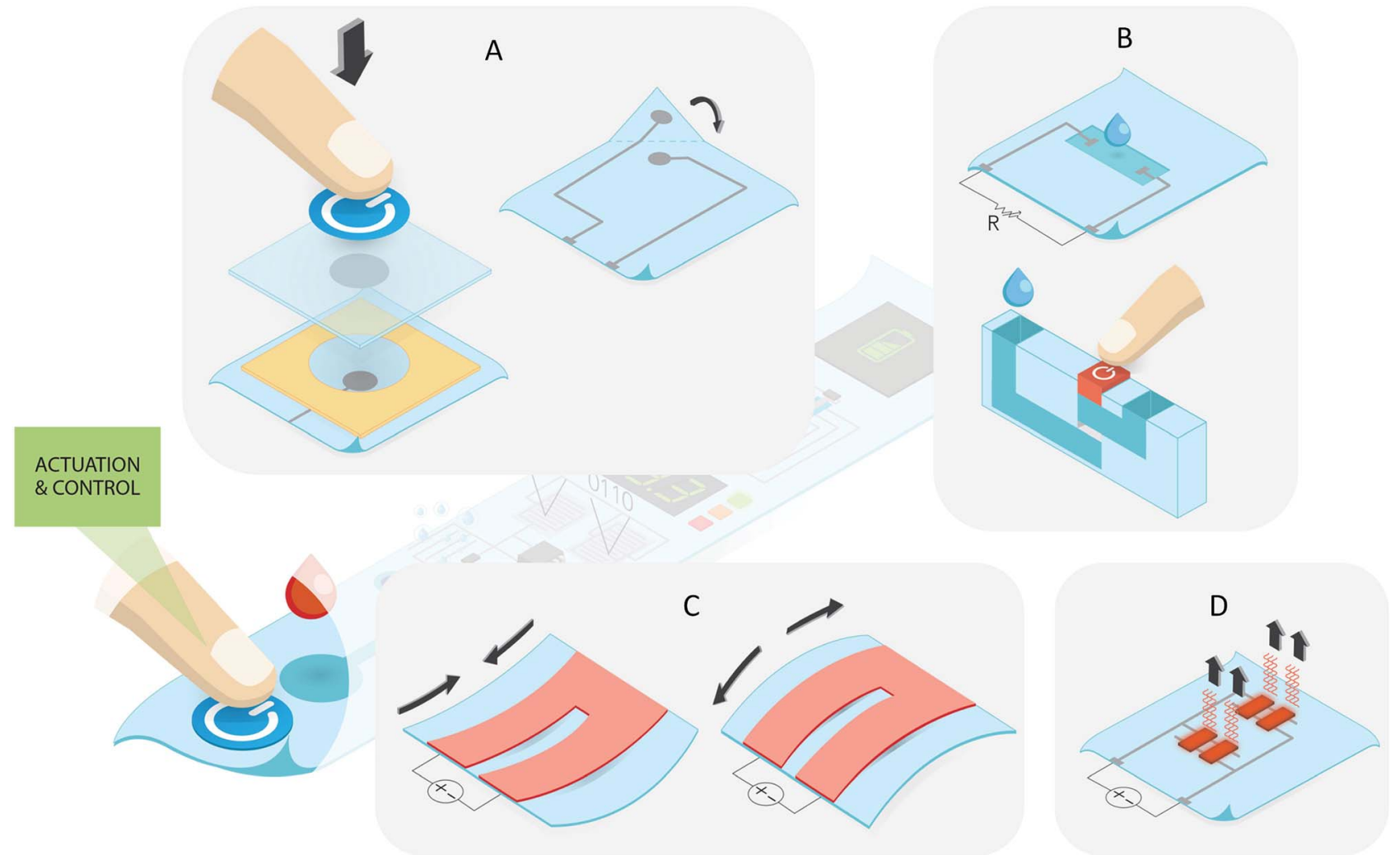

Fig. 5 Paper-based functional components for actuation and control. Switches and valves are illustrated for both (A) electronic and (B) fluidic set ups. (A) Paper-based push buttons, which when pressed, compress a spacer, create contact and close a switch. Fold-over paper designs to make electrical connections and close a switch can also be used. (B) For fluidic implementations, ionic fluids can be introduced to create an electrical pathway, closing a switch, and three-dimensional stacked paper structures with spacers, fluidic channels and buttons can be used to implement paper-based fluidic switches, with a cross-sectional view illustrating this. (C) Movement can be implemented through printing of functional inks which when subjected to voltage and temperature changes, undergo compression or expansion to create motion. (D) Heating on paper using positive temperature coefficient (PTC) inks. These functional inks increase in temperature in response to an applied voltage and self-regulate at a designed temperature for constant and controlled heating.

\subsection{Electrochemical sensors}

Electrochemistry is a powerful tool for health and environmental diagnostics, and various paper-based electrochemical sensors have been explored. ${ }^{\mathbf{6 4 , 6 5}}$ Electrochemical sensors typically consist of a working electrode (WE), counter electrode (CE) and reference electrode (RE). WE and CE are often implemented using carbon-based materials (Fig. 6A), including graphenebased pastes. ${ }^{6}$ Simple screen printing techniques are often used to implement electrochemical sensors directly on to paperbased diagnostic tests. Materials development for printed and wearable electrochemical sensors have seen extensive growth, ${ }^{7}$ with examples for diagnostic applications ranging from water quality monitoring ${ }^{67}$ to cancer detection. ${ }^{68}$

Paper-based electrochemical sensors have been successfully implemented for detection of glucose, diabetes markers total and glycated haemoglobin (HbA1c) and heavy metals in serum, ${ }^{69}$ showing high sensitivity within clinically relevant ranges. Stability, accuracy and shelf life of paper-based electrochemical sensors need to be explored but show promise as effective on-board sensors for ASSURED paper-based diagnostics. However, these sensors require electrochemical analysis instrumentation, currently implemented using portable potentiostat systems or smart phones. In future these could be replaced by printed electronic processing components, with an example of a hybrid printed, paper-based potentiostat recently showcased. ${ }^{70}$ These could be integrated into ASSURED devices in the long-term, along with printed read out components to display the result.

\subsection{Temperature, humidity and gas sensors}

Measurement of environmental parameters plays an important role in paper-based diagnostics. Printed temperature sensors or thermometers consist of temperature sensitive printed resistors, where changes in temperature cause variations in the resistance (Fig. 6B). Examples using both inkjet and screen printing on to various flexible substrates including polyethylene terephthalate (PET), paper and fabric have been presented. ${ }^{61}$ Temperature sensitive luminescence indicators with laminated paper devices have also been illustrated to give visual temperature readouts. ${ }^{71}$ Commercial printed temperature sensor examples on PET substrates by Thinfilm in conjunction with Printed Sensor Technologies (PST) ${ }^{72}$ use novel silicone inks and show high reliability. 


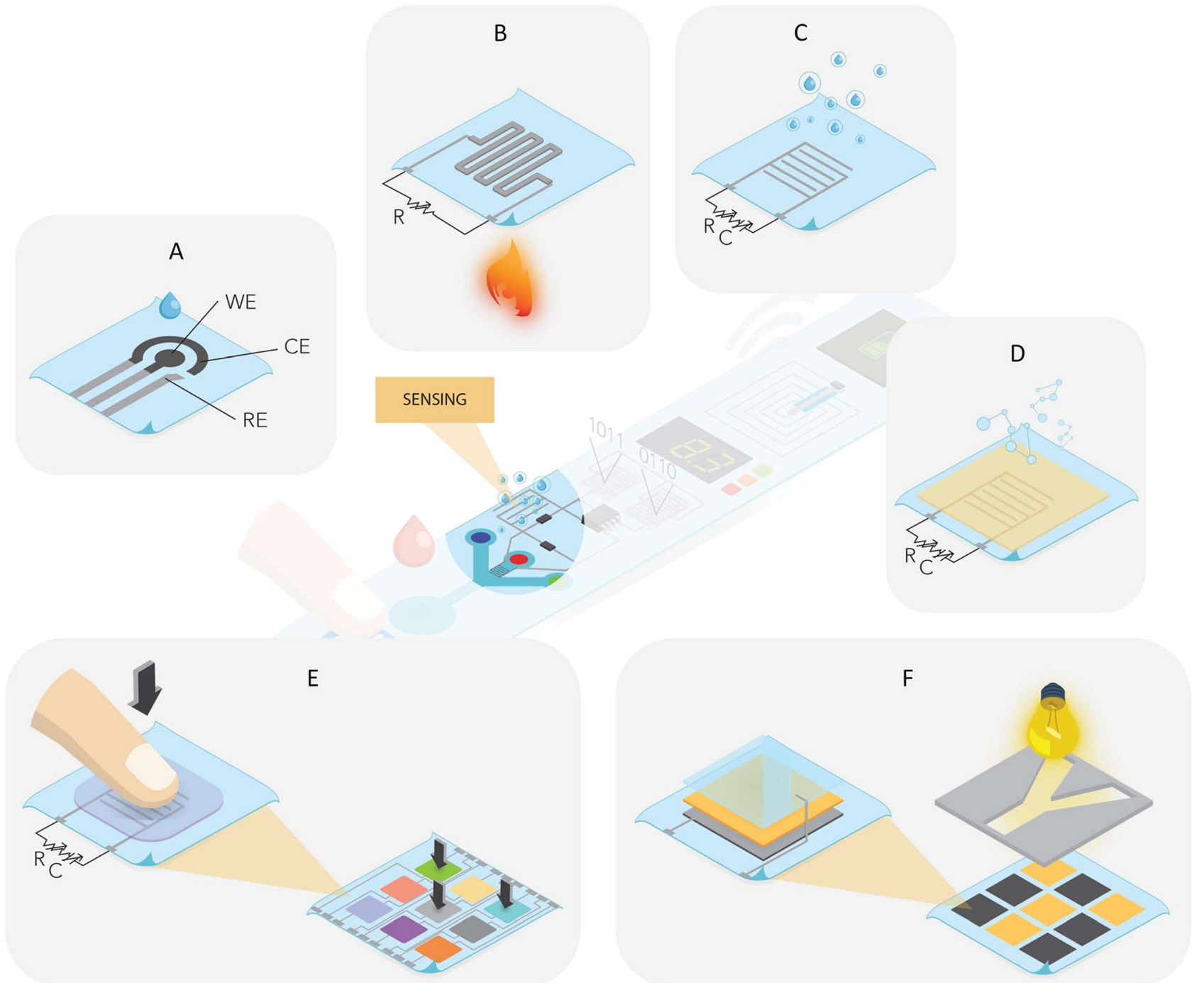

Fig. 6 Printed and paper-based sensing functional modules. (A) electrochemical sensors with working electrode (WE), counter electrode (CE) and reference electrodes (RE) indicated. Conductive materials are used for the connections and RE, while WE and CE consist of printed electrochemical functional materials. (B) Temperature sensors, with printed conductive materials and printed temperature sensitive material which varies in resistance as the temperature changes. (C) Humidity sensors, utilizing printed conductive tracks typically in an interdigitated format, which vary in resistance or capacitance as the moisture content changes. (D) Gas sensors, with a similar structure to humidity sensors but with a printed gas sensing material coating the conductive tracks. (E) Pressure, touch and proximity sensors, typically with printed pressure sensitive material over conductive tracks which varies with resistance and/or capacitance in response to pressure. These individual sensors can be implemented in matrix format to realize touch sensors. (F) Light sensors with a printed conductive electrode (bottom), printed photosensitive material (middle) and printed transparent electrode (top). These photodetector elements can also be implemented in matrix format to realize image sensors.

Different types of humidity sensors include resistive, colorimetric, capacitive or surface acoustic wave (SAW) ${ }^{73}$ Capacitive type sensors are linear, require less complex circuitry and can operate over a wide range of humidity. These sensors detect a change in relative permittivity of the surrounding medium, where an increase in capacitance indicates an increase in moisture content ${ }^{27}$ (Fig. 6C). Different capacitor structures such as parallel plates, meshes and interdigitated designs can be utilized for humidity sensing. Successful printing of humidity sensors on to cardboard and recycled substrates has been demonstrated ${ }^{73}$ and a paper-based electrical respiration sensor based on moisture content serves as a recent example with direct point-of-care applications. ${ }^{74}$ Paper-based humidity sensors for effective detection in the range of $20-90 \% \mathrm{rH}$ and with lifetimes of up to 4 months have been demonstrated, ${ }^{75}$ showing potential for direct integration into ASSURED devices.

Gas sensors were first focussed on humidity sensing, but have been expanded through the development of printable gas sensitive films. ${ }^{61}$ Flexible gas sensors (Fig. 6D) typically utilize resistive or capacitive sensors printed using specialized materials such as semi-conductive nanoparticle inks and solid electrolyte inks. ${ }^{27}$ Examples include resistive nitrogen oxide gas sensors, capacitive hydrogen gas sensors, carbon monoxide and oxygen sensors. 
A flexible ammonia gas $\left(\mathrm{NH}_{3}\right)$ sensor on photo paper showed high stability over several months ${ }^{76}$ while an inkjet printed paper-based wireless humidity and hydrogen sulphide gas $\left(\mathrm{H}_{2} \mathrm{~S}\right)$ sensor ${ }^{77}$ illustrated the feasibility of incorporating various passive, wireless sensors into an integrated paper-based device.

\subsection{Pressure, touch and proximity sensors}

These sensors are applicable to paper-based diagnostics for detection of environmental surroundings, device handling and for user interaction. Similar to humidity sensing, available printed touch sensor technologies include capacitive, resistive, optical touch, acoustic wave, infrared, and others, each suited to different applications. ${ }^{27}$ The low cost of implementing resistive sensors makes this a favourable technology, followed by capacitive sensing. Piezoelectric papers have been developed for force and pressure sensing applications, ${ }^{55}$ and are also discussed in Section 4. Examples such as graphite paper-based piezo-resistive sensors ${ }^{78}$ and touch and proximity sensors printed on paper-based substrates ${ }^{79}$ show promise for ease of implementation and integration with paper-based diagnostics.

Wearable pressure sensors realized using gold nanowires on tissue paper have shown high sensitivity for blood pulse monitoring, as well as for detecting the weight distribution of small objects across an array of pressure sensors. ${ }^{80}$ Printed pressure sensitive inks are commercially available, and can be used for large area pressure sensing in various form factors. ${ }^{5}$ The resistance of the ink varies with the force applied to the printed ink surface with a common design approach making use of interdigitated electrodes as a base layer with the pressure sensitive material printed over the top (Fig. 6E).

A paper-based $5 \times 5$ bimodal sensor has recently been demonstrated for temperature and pressure sensing ${ }^{\mathbf{8 1}}$ using conductive inks and carbon nanotubes. Temperature sensing capabilities were demonstrated over a range of $150{ }^{\circ} \mathrm{C}$, along with high pressure sensitivity over a range of $100 \mathrm{~Pa}$ to $5 \mathrm{kPa}$ with high endurance characteristics. The application proposed is for electronic skin, and shows promise for ease of integration with ASSURED paper-based devices to achieve various sensing capabilities in a single, paper-based device.

\subsection{Light and image sensors}

Printable light sensors or photodetectors have recently been developed $^{\mathbf{8 2 , 8 3}}$ and are typically realized by an organic (or less commonly inorganic) photoactive layer between two transparent electrodes $^{61}$ (Fig. 6F). Applications of printed photodetectors include industrial and medical large-area sensing, particularly for colour detection, with potential application to colorimetric detection implemented on paper-based diagnostic devices. In addition, security features for confidential diagnostic testing could potentially be implemented through finger print imaging which could be integrated as part of future envisaged paper-based diagnostic devices.

Photodetectors printed on to paper have been demonstrated, ${ }^{\mathbf{8 4}}$ along with inkjet-printed photodiodes, ${ }^{\mathbf{8 5}, 86} \mathrm{UV}$ photodetectors and infrared sensors. ${ }^{62}$ An overview of the components and integration of these in to a functional flexible image sensor have also been showcased, ${ }^{87,88}$ consisting of photodiodes integrated with organic thin film transistors in an active matrix (Fig. 6F). This technology is in the process of being commercialized $^{89}$ for a sensor with a $4 \mathrm{~cm} \times 4 \mathrm{~cm}$ active area. ${ }^{90}$

A low-cost, broadband, paper-based photodetector using ZnS- $\mathrm{MoS}_{2}$ has recently been presented. ${ }^{91}$ The demonstrated sensitivity over a broad spectrum shows promise for development of large-scale, low-cost paper-based photodetectors. These devices could be utilized in integrated ASSURED devices for colorimetric readout of paper-based microfluidic results.

\section{Data processing and storage}

Once detection has taken place, automated and accurate analysis would be ideal for paper-based diagnostics to provide a quantitative, error-free result without requiring user skills or training. Printed components for computational functions are thus required, and both hybrid (Section 3.1) and fully printed solutions have been explored. Hybrid printed or paper-based solutions using existing packaged integrated circuits or die formats of integrated circuits enable the same processing capabilities to be achieved as with traditional electronics, but connection issues between components and flexible paper substrates can pose challenges.

\subsection{Integrated circuits}

Processing and amplification stages have been implemented through printed high gain amplifiers and digital-to-analogue (DAC) converters, ${ }^{34}$ as well as ring oscillator circuits. ${ }^{92}$ Printed programmable logic circuits have been realized using transistor arrays $^{93}$ (Fig. 7A) to demonstrate a ring oscillator, a D-type flipflop memory element, a demultiplexer and a programmable array logic device. Medium-scale integrated circuits consisting of 100 transistors have been showcased, ${ }^{94}$ as well as inkjetprinted thin film microprocessors ${ }^{95}$ which can be programmed after manufacture via inkjet printing. Successful printed ring-oscillators in the range of $\mathrm{kHz}$ have been demonstrated, showing promise for complex analogue-to-digital converters (ADCs). ${ }^{\mathbf{9 6}}$

The development of paper-based transistors, as discussed in Section 3.2.4, will be key in the advances of paper-based integrated circuits. Robust and fast paper-based transistors will enable integrated circuits to be implemented for processing in ASSURED devices, and could be operated using on-board printed energy sources if low operation voltages are achieved.

\subsection{Memory components}

Data storage requirements are an important consideration for sensor data and user input logging. Printed memory functionality has been demonstrated, commonly by applying a current to keep a stored memory state in a transistor. Printed memristors undergo resistance changes in response to an applied current, only requiring a current to change the state, not to keep it, as in the case of a transistor. Memristors are thus advantageous as printed memory components as they are more energy efficient and physically smaller than transistors. Memristors 


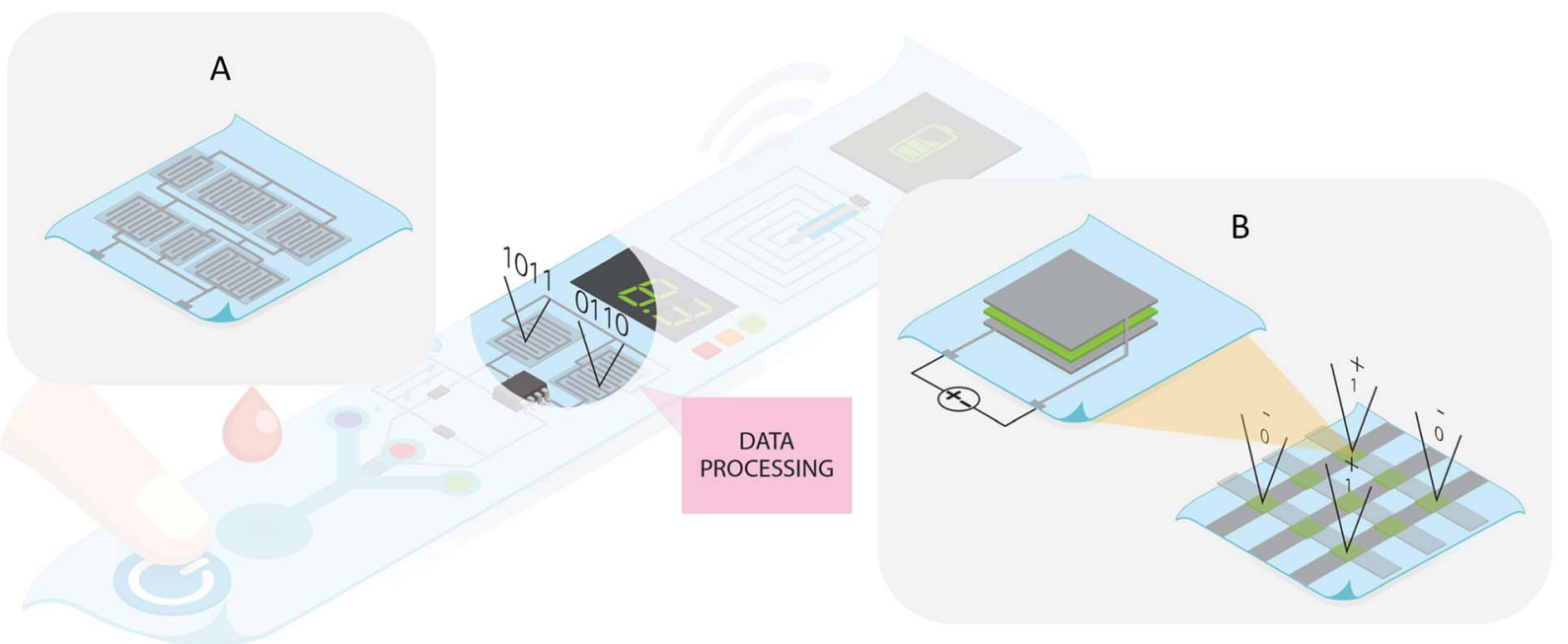

Fig. 7 Printed data processing and storage functionality. (A) Printed integrated circuits and processors, typically constructed from various printed transistor combinations. (B) Memory components, with individual printed units constructed from printed conductive electrodes with a resistive switching material in between. When implemented in a matrix format, a printed conductive electrode matrix can be implemented, with individual printed resistive switching pixels which can each be set to 1 or 0 values by applying positive or negative voltages, respectively.

consist of two conductive electrodes with a thin layer of memristive material (typically a titanium oxide derivative) between them and have been inkjet printed on plastic substrates successfully. ${ }^{97}$

Write once read many (WORM) memory has also been implemented in printed form ${ }^{98}$ to allow for more complex memory functionality, including moderate bit counts and read and write functionality. ${ }^{90}$ Inkjet printing and large scale rollto-roll fabrication using gravure and flexography methods have been implemented successfully for this technology. ${ }^{27}$ These developments show promise for complex printed integrated circuits to be produced in large volumes and become commercially implemented. Rewritable resistive switching techniques for printed memory have been showcased, ${ }^{99}$ with a number of paper-based memory devices demonstrated ${ }^{\mathbf{1 0 0 , 1 0 1}}$ (Fig. 7B).

Paper-based, foldable and disposable memory with a low voltage operation of $1.5 \mathrm{~V}$ has been shown, ${ }^{101}$ which would allow for on-board battery operation if integrated as part of an ASSURED paper-based device. Additionally, memory performance was maintained up to $100 \mathrm{~s}$ of switching cycles after folding tests were carried out, showing robust and repeatable characteristics that align well with the ASSURED principles. Advances in printing on to paper substrates to improve conductivity, foldability and disposability, ${ }^{\mathbf{1 0 2}}$ will drive effective printed, paper-based data processing and storage systems.

\section{Read out and display}

Result read-out is important to provide the user with feedback from a diagnostic test and to digitize the result for further communication, processing and storage. Various current printed display technologies ${ }^{\mathbf{1 0 3 , 1 0 4}}$ can be explored to fulfill this role.

\subsection{Paper-based microfluidic visual indicators}

Displays that are built in to the paper-based test device itself through chemical reactions and biological processes can be utilized (Fig. 8A). Detection principles such as chemiluminescence, where light is generated based on a chemical reaction, have also successfully been showcased. These techniques are advantageous as they do not require an excitation source, as in the case of fluorescence. Comprehensive reviews of read-out and display techniques implemented for paper-based diagnostics summarize recent developments in this field., ${ }^{2,3}$ These techniques have been successfully integrated with paperbased diagnostics towards fully ASSURED solutions, such as a commercial example by haemokinesis105 for blood typing using visual text readout generated from the sample.

\subsection{Thermochromic displays}

Thermochromic readouts were some of the earliest examples of displays on paper-based platforms ${ }^{\mathbf{1 0 6}}$ (Fig. 8B). Seven-segment displays on photopaper ${ }^{\mathbf{1 0 7}}$ and successful integration with paper-based diagnostics ${ }^{2}$ using thermochromic techniques have been demonstrated. Thermochromic ink is patterned on to one side of a paper substrate with conducting wires on the opposite, so that when a current is passed through the wires, the thermochromic ink is heated and causes a colour change which is usually reversible. Visual graphics using these techniques are advantageous over text to maximize successful user interpretation, particularly in multilingual or illiterate environments. Although these solutions utilize low-cost materials, analytical applications using this technology have not yet been demonstrated, likely as a result of the high currents $(>25 \mathrm{~mA})$ typically necessary for visible changes in thermochromic displays to occur. ${ }^{2}$ In addition, environmental temperature plays a role in device functionality and will need to be 

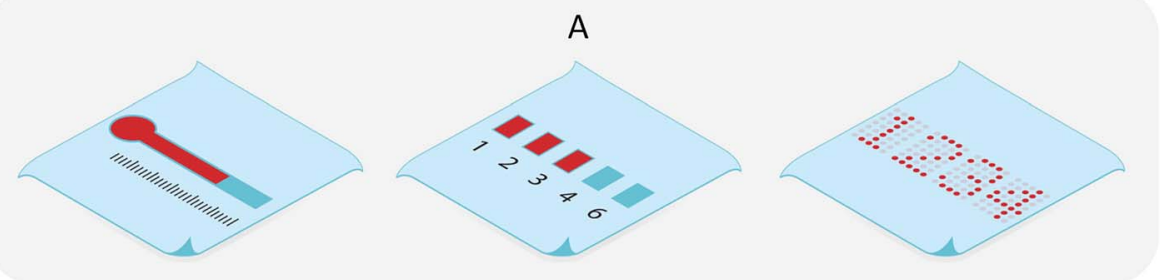

B

.

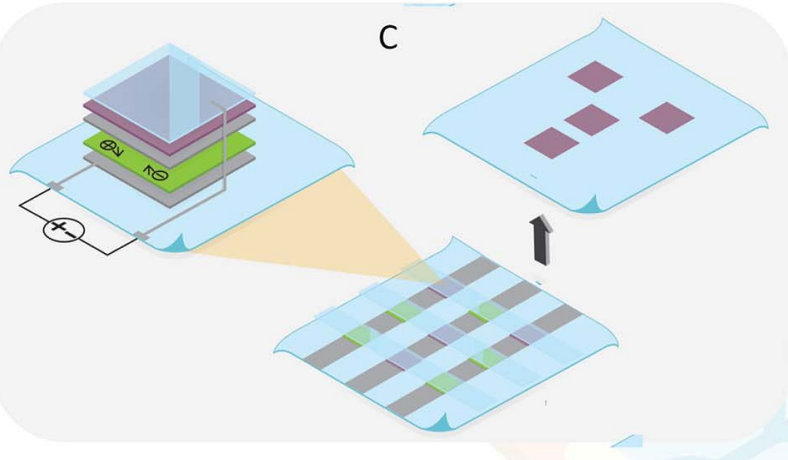

$E$
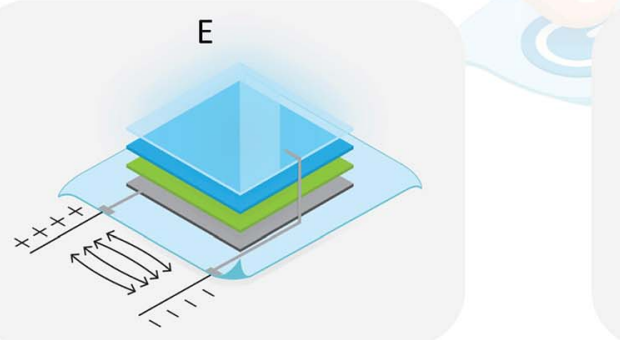
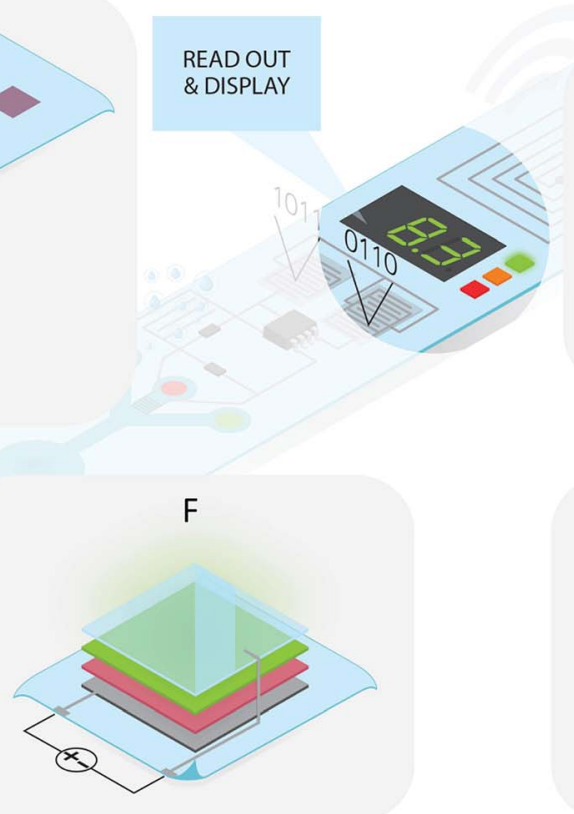

READ OUT
\& DISPLAY
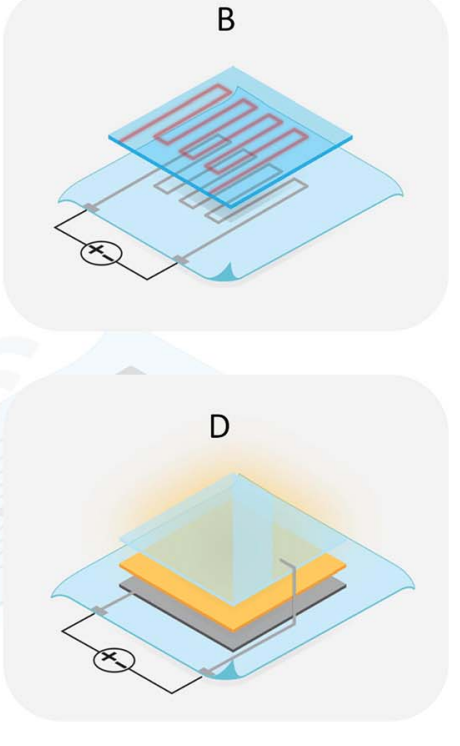

Fig. 8 Printed and paper-based read out and display module examples. (A) Paper-based microfluidic visual indicators, which can be distance or time based, counting based or text based, utilizing the sample and resulting flow or reactions to provide user feedback on the test progress and results. (B) Printed thermochromic displays where applying a voltage to conductive elements covered by a printed thermochromic material results in a colour change in response to a temperature increase. (C) Printed electrochromic displays, with individual pixels made up of printed layers on top of paper as follows: conductive electrode, electroactive material, electrolyte, electrochromic material, and a transparent electrode on top. Applying a voltage causes the electrochromic material to change colour. Pixels can be implemented in matrix format to enable effective text displays. (D) Printed light-emitting electrochemical cells consist of an active material between two electrodes, with the top electrode (cathode) being transparent. Light is emitted by the active material in response to a voltage applied across the electrodes. (E) Electroluminescent displays, with individual units consisting of a conductive electrode on the paper substrate, followed by a printed dielectric layer, a phosphor material and a top transparent electrode. Light is emitted in response to an electric field. (F) Organic light-emitting diodes (OLEDs) consist of two electrodes with a conductive material and an emissive electroluminescent material in between. The latter emits light through the top transparent electrode in response to a current applied at the electrodes. (G) Electrophoretic displays consist of charged black and white particles that change orientation in response to an applied electric field.

investigated if successful integration with paper-based ASSURED devices is to be realized.

\subsection{Electrochromic displays}

Electrochromic displays (ECDs) operate similarly to thermochromic displays by converting an electrical signal in to an optical readout. A colour change occurs when an electron is gained or lost as a result of an applied current or potential. Reversing the polarity of the voltage causes the material to change back to its original colour. The electrochromic material must be in contact with the electrode and the electrolyte layer to exchange both electrons and ions, respectively (Fig. 8C). Alternatively, the electrochromic material can be dispersed in the electrolyte layer. ECDs can be operated in transmittance or reflectance mode, the latter being likely for paper-based implementations. Colour is controlled by the amount of voltage and polarity applied to the electrode terminals ${ }^{27}$ enabling matrix addressed ECD displays to be implemented ${ }^{108-110}$ (Fig. 8C). Commercialization of printed ECDs has been explored by Ynvisible, Acreo and NTera. ${ }^{27}$

Electrochromic read-out in an integrated paper-based device with a paper battery and electrochemical sensor using Prussian blue has been showcased, ${ }^{111}$ with a more recent example to visually represent changes in resistive sensors. ${ }^{112}$ The system enables semi-quantitative readout of voltage or resistance changes produced in the circuit, ranging from $-0.5 \mathrm{~V}$ to $-2 \mathrm{~V}$ for distance and counting based implementations. The limited detectable resolution could be further explored, but already shows promise for applications towards fully integrated paper-based ASSURED devices. 


\subsection{Light emitting electrochemical cells}

Flexible, lightweight display modules on paper substrates can be effectively implemented using light emitting electrochemical cells (LECs). LECs are typically made up of an anode, cathode and active material which generates light when a voltage is applied (Fig. 8D). Low operational voltage requirements and airstable electrodes make LECs power efficient with simplified encapsulation requirements. ${ }^{\mathbf{1 1 3}}$ These advantages in addition to the compatibility of LECs with paper substrates make this a promising emerging display technology for low-cost, paperbased point-of-care diagnostics.

Different types of paper have been utilized, including standard printing paper, where the fabrication of LECs did not require specialized processing. ${ }^{\mathbf{1 1 4}}$ A light emitting textile device using LEC technology has also been presented, ${ }^{115}$ with potential applications to fibrous paper-based diagnostic substrates. Recent advances have resulted in multi-coloured LEC fabrication towards low-cost light sources with high luminance. ${ }^{116}$ Voltages required were approximately $3 \mathrm{~V}$, enabling effective lighting that could be achieved using battery power to realize visual indicators on ASSURED devices. A recent example of biocompatible LECs $^{\mathbf{1 1 7}}$ contributes to the long-term goal of disposable and environmentally friendly ASSURED devices.

\subsection{Electroluminescent displays}

Electroluminescence enables a material to emit light in response to an electrical current or a strong electrical field. Electroluminescent displays (ELDs) consist of one or more layers of inorganic phosphor and insulator stacks sandwiched between two electrodes (an anode and cathode) that emit light when exposed to an electric field (Fig. 8E). Different types of electroluminescent displays include powder or thick-flim displays, thin-film displays, and hybrids of these. Powder electroluminescent displays have been successfully fabricated on different paper substrates, including glossy, sticker, newspaper and magazine papers. ${ }^{118}$ These devices showed satisfactory luminance values for realizing clear visual illumination on lowcost paper substrates. Commercial electroluminescent displays can provide uniform light over relatively large areas and can be manufactured in to thin structures using power efficiently. ${ }^{\mathbf{1 0 4}}$ Although electroluminescent displays usually require small amounts of current to operate, high driving voltages are required, which could be challenging to implement for standalone, integrated ASSURED devices.

\subsection{Electrochemiluminescent displays}

Electrochemiluminescence (ECL) is a derivative of chemiluminescence, where the signal is generated from an electrochemical reaction. Electrochemiluminescent read out thus offers a higher degree of control by the user than purely reaction-based chemiluminescence, but requires power to apply a potential. Existing examples of electrochemiluminescence in paper-based diagnostics usually require external instrumentation to apply voltages, and to read out and quantify the emitted signal. ${ }^{2}$ Various power supply methods, including thermal- powered, self-powered, wireless and light-powered methods including digital cameras and smartphones can be implemented as discussed in recent reviews. ${ }^{119,120}$ ECL readout in paper-based microfluidic devices have been successfully implemented for detection of DNA, proteins and cancer cells, for example, and have utilized smart phones for powering the reaction and detecting the result using built-in cameras. ${ }^{119}$ This shows promise for integration of ECL detection and readout on ASSURED paper-based diagnostics, with low power requirements for ease of visual readout being feasible.

\subsection{Organic light emitting diodes (OLEDs)}

OLEDs enable flexible and printable lighting and display solutions. ${ }^{\mathbf{1 0 4}}$ A layer of organic material is sandwiched between two conductors (an anode and a cathode) (Fig. 8F). The organic layer of emissive electroluminescent enables bright red, green and blue light to be emitted in response to an electric current. ${ }^{27}$ OLEDs differ from ELDs in that the light emitting material is organic, compared with the inorganic phosphors in ELDs. Inkjet printing has enabled mass production of flexible OLED devices through fast deposition of organic material without further patterning required. Encapsulation for protection from moisture and oxygen are important design considerations in the printing of OLED displays. ${ }^{27}$ The development of paperbased OLEDs has been limited as a result of the high sensitivity of OLED active materials requiring exact thicknesses, which can be difficult to achieve on paper, ${ }^{114}$ but functional, flexible OLEDs fabricated using standard printing paper ${ }^{\mathbf{1 2 1}}$ and transparent cellulose substrates ${ }^{\mathbf{1 2 2}}$ have been presented. OLEDs manufactured on cellulose exhibited constant current and power efficiencies in ranges of 10 to $100 \mu \mathrm{A}$ and 4 to $8 \mathrm{~V}$. These devices could be integrated as part of low-cost, flexible ASSURED paper-based diagnostics for visual feedback or result readout, making use of on-board battery power and an environmentally friendly approach.

\subsection{Electrophoretic displays}

Electrophoretic materials form shapes by rearranging charged pigment particles using an electric field, as opposed to emitting light. Positively and negatively charged black and white particles that are bistable are suspended in liquid and allow for print quality resolution to be achieved. ${ }^{104}$ (Fig. 8G) Electrophoretic displays (EPDs) are found in e-book readers, watches and other electronic displays with additional colours recently becoming available. ${ }^{123}$ EPDs only consume power when the display is being updated and provide high quality readability regardless of lighting conditions, and are thus well-suited for point-of-care diagnostics in under-resourced settings. Although EPDs are commercially available, they have not been implemented directly on to paper substrates and thus integration of this technology with paper-based diagnostics has not yet been explored.

A merge between fluidics and display technologies has been shown through electrowetting displays on paper, ${ }^{\mathbf{1 2 4}}$ and electrofluidic displays. ${ }^{125}$ Advances in organic light emitting transistors (OLETs) that combine the light emitting capabilities of 
an OLED with the switching capabilities of transistors show promise as a printable display applicable to various substrates, including paper. $^{27}$

\section{Connectivity}

Communication of the result from the paper-based diagnostic device will be required for effective result recording and filing of information towards an integrated solution. Connections between paper devices and external instrumentation can be achieved through physical connectors and cables, including disposable USB connectors on paper ${ }^{126}$ (Fig. 9A).

Wireless communication technologies are particularly advantageous for under-resourced settings, as they eliminate the need for physical connections to instrumentation and prevent contamination. The evolution of the internet of things (IoT) has resulted in a drive to include connectivity between devices and systems. ${ }^{127}$ Various wireless technologies can provide connectivity for paper-based diagnostic devices, with passive technologies such as radio-frequency identification (RFID) being attractive options as these are low-power, printable, low-cost, and thus ideally suited to paper-based diagnostic devices. Other technologies include near field communication (NFC), Bluetooth, WiFi, ZigBee, Infrared, and cellular technologies such as $3 \mathrm{G}$ and $4 \mathrm{G},{ }^{128}$ each making use of different standards, frequency ranges and operational distance ranges. Passive short range communication technologies such as RFID and NFC are better suited from a low-cost, disposable device perspective, where power is not required on the device itself, and have been applied to point-of-care solutions for implantable, wearable and hand-held wireless biosensors. ${ }^{\mathbf{1 0 2 , 1 2 9}}$ RFID technologies have also been highlighted as beneficial solutions for rural e-Health systems ${ }^{\mathbf{1 3 0}}$ and have been used in hospitals for patient monitoring with some success. ${ }^{\mathbf{1 3 1}}$ Recently, privacy preservation in RFID systems for healthcare has also been pursued, ${ }^{\mathbf{1 3 2}}$ to enable a secure, confidential method of data communication and storage.

RFID is well-suited to paper-based diagnostics as the tag antennas are printable and paper is well-suited for ultra-high frequency (UHF) and microwave applications. ${ }^{133}$ Printing of antennas ${ }^{133-137}$ and specifically RFID tags ${ }^{138-143}$ on to low-cost paper and cardboard substrates has been investigated using both inkjet and screen printing techniques (Fig. 9B). Combinations of RF, microfluidics and inkjet printing technologies to implement wireless sensing platforms that are activated by fluids in microfluidic channels have also been studied. ${ }^{\mathbf{1 4 4 , 1 4 5}}$ Fluids can be used to activate or control the properties of the antennas.

Sensing RFID systems have evolved in recent years, ${ }^{\mathbf{1 4 6}}$ adding to the value proposition of RFID technologies for integrated sensing and connectivity of paper-based diagnostics. Some paper-based communication and sensing modules that have been implemented include patch antennas, UHF oscillators, mixers and frequency doublers, ${ }^{\mathbf{1 4 7}}$ which make up a variety of communication capabilities. In addition, paperbased microfluidics have been integrated with selfassembling RFID tags to create a self-powered biosensing platform. ${ }^{148}$ Commercially available NFC RFID tags embedded in to paper substrates are also available ${ }^{\mathbf{1 4 9}}$ for various applications. These technologies can be integrated with paperbased ASSURED devices in their current form to enable effective wireless communication of information and results from the disposable diagnostic devices for processing and storage.

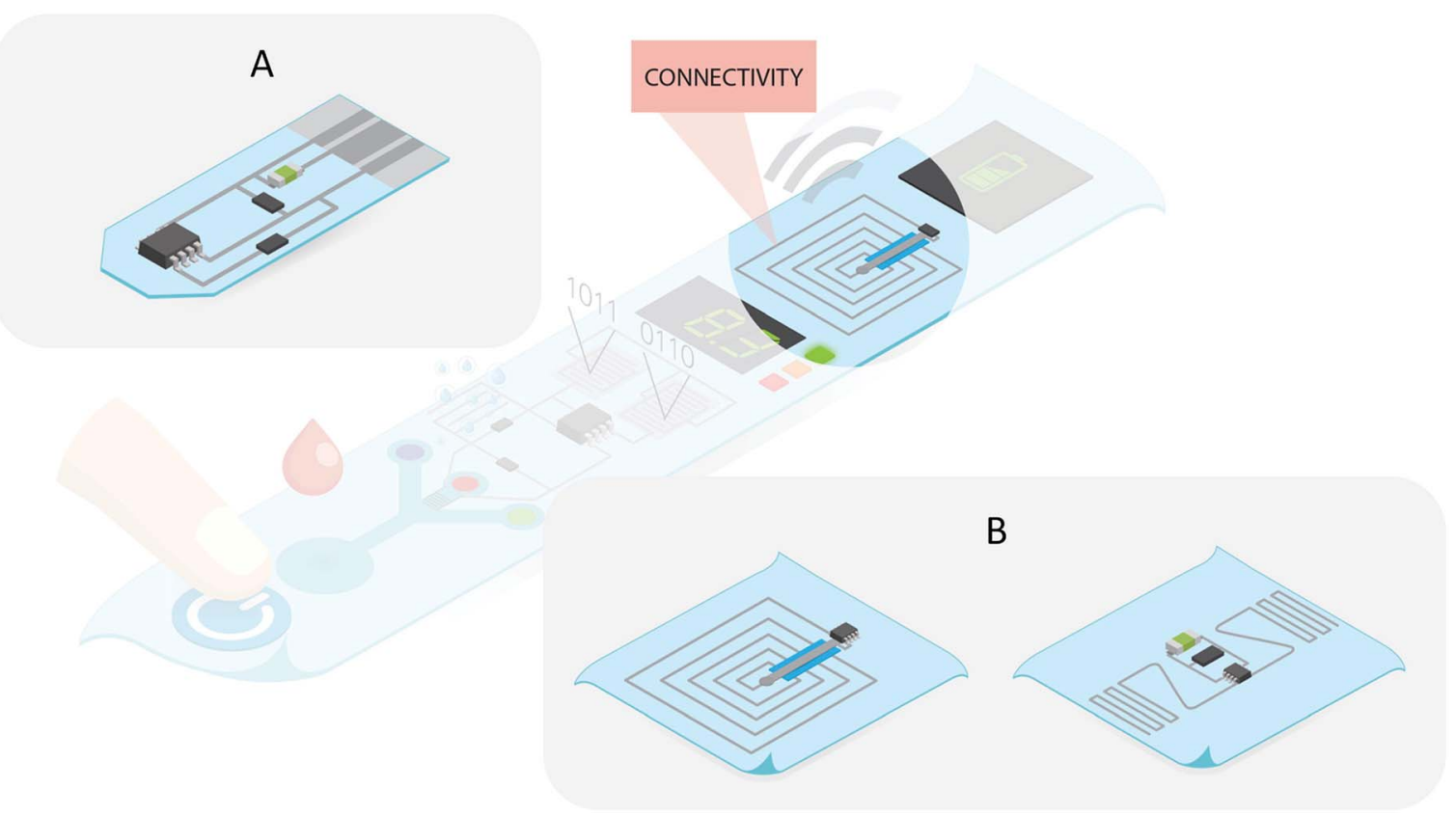

Fig. 9 Printed paper-based connectivity modules. (A) Physical printed connectors on a paper-based electronic device to connect to standard ports or adapters. (B) Wireless connectivity through printed NFC and RFID tags, which can also be integrated with microfluidics. 


\section{Energy storage}

Where wireless power transmission will not suffice to drive the various components of integrated ASSURED paper-based diagnostic devices, built-in power will be required. Printed energy storage developments have led to some commercial examples as well as integration with paper-based diagnostics, with a number of reviews highlighting the progress made, ${ }^{150-153}$ along with various integration considerations. ${ }^{154}$

\subsection{Batteries}

A typical paper-based battery consists of two electrodes made from metal or carbon deposited on to paper substrates, with an electrolyte-filled paper in between to connect the two electrodes by a salt bridge or ion exchange membrane (Fig. 10A and B), and demonstrates the usefulness of paper in these devices. ${ }^{26}$ Foldable or stacked paper designs with wax patterned wells for the various reagents have been implemented ${ }^{155,156}$ with higher porosity papers tending to yield higher current densities. Printed batteries can either be in sandwich (Fig. 10A) or parallel (Fig. 10B) formats, with several printing techniques having been utilized $^{151}$ to realize customized batteries. ${ }^{157}$ Commercially available printed primary (non-rechargeable) batteries have been under development for a few decades, commonly utilizing the well-known manganese dioxide/zinc battery chemistries and with scale up possible for roll-to-roll manufacture. ${ }^{27}$ Printed secondary (rechargeable) batteries are being explored with potential applications in wearable electronics. ${ }^{158}$

Lithium ion batteries are well suited to applications requiring high power and energy densities and show promise as rechargeable and durable power sources. ${ }^{150}$ Ions are transported between the anode and cathode through an ion-conductive electrolyte that is electronically insulating. Typical achievable voltages are around $3 \mathrm{~V}$ with energy capacities of $2250 \mathrm{~mA} \mathrm{~h}{ }^{159}$ Paper-based lithium ion batteries typically make use of commercial papers that are coated with carbon nanotubes to create highly conductive, easily manufacturable battery components ${ }^{160,161}$ which can be foldable. ${ }^{162}$

Paper-based lithium-ion batteries can generate power a few orders of magnitude larger than in paper-based electrochemical batteries or biofuel cells, but require multiple layers of materials and stringent packaging requirements. These cost implications and concerns of environmental friendliness could limit integration with existing paper-based electronics and diagnostic devices, particularly in terms of cost and disposability, but could be well-suited to polymer-based flexible electronic applications. ${ }^{150}$

Recent examples of paper-based batteries enable sufficient powering of LEDs and handheld devices such as glucometers, ${ }^{163}$ which could be used to drive various sensors and visual readout or displays for paper-based ASSURED diagnostics.

\subsection{Biofuel cells}

Biofuel cells utilize mircoorganisms or enzymes as catalysts to convert fuel such as ethanol and water in to power, instead of using metal catalysts as in the case of electrochemical batteries. ${ }^{26}$ Biofuel cells are thus low in cost, renewable and environmentally friendly. Microbial fuel cells usually have long lifetimes, but lower power densities, while enzymatic biofuel cells have higher power densities but limited lifetimes of a few days or weeks as a result of enzyme fragility. ${ }^{150,159,164}$ Typical power densities for microfluidic enzymatic biofuel cells have been reported to be several orders of magnitude higher than those achieved for microbial fuel cells, ${ }^{159}$ with comparisons made for implantable devices. ${ }^{165}$

Fuel cells implemented on lateral flow devices have been investigated ${ }^{166}$ (Fig. 10C), as well as bacteria-driven paper-based biofuel batteries, ${ }^{167,168}$ capable of powering an LED for up to 30 min. Low-cost (less than $\$ 1$ per device) paper-based microbial fuel cells utilizing a drop of sewage as fuel have been presented ${ }^{169}$ with a more recent stackable design developed. ${ }^{170}$

Examples of recently developed paper-based enzymatic fuel cells are capable of illuminating LEDs ${ }^{171}$ or powering a digital clock for 3 days. ${ }^{172}$ The current capabilities of these fuel cells enable them to be used for read-out and display modules for paper-based ASSURED devices, including low power displays such as EPDs or individual LED indicators, and offer an environmentally friendly solution.

\subsection{Supercapacitors}

Supercapacitors are electrochemical energy storage components and are similar to batteries in terms of design, manufacture and structural dimensions, consisting of two electrodes that are electrically isolated by a separator with an electrolyte. When a potential is applied, an electric field is formed over the interfaces between the electrode and electrolyte. The energy is stored in the electric field in a way that is comparable to conventional capacitors. Supercapacitors typically have lower energy densities than batteries, but higher power densities, with faster charge/discharge rates, as well as being considered more environmentally friendly and safer than batteries. An in-depth review on the various aspects of supercapacitors provides further details. ${ }^{173}$

Supercapacitors have been implemented on standard office papers with carbon nanotubes and ionic-liquid-based gel electrolytes, ${ }^{174}$ as well as on paper substrates using polyanilinebased supercapacitors. ${ }^{175}$ Commercial pencils and office paper have been utilized to realize interdigitated electrodes ${ }^{176,177}$ (Fig. 10D) with supercapacitors implemented in both a pouch format with paper separators and liquid electrolyte, as well as with a solid electrolyte. Various form factors and aesthetic designs have been utilized using this approach. ${ }^{178}$ A photoelectrical origami device with an internal chemiluminescent light source and an integrated paper supercapacitor was developed to collect and store generated photocurrents. ${ }^{179}$ Printed supercapacitors have found uses for memory back-ups, as well as in packaging and RFID systems. ${ }^{27}$ The high current/ power generation possible makes these devices an attractive energy storage solution.

A recent example of all-printed paper-based supercapacitors, fabricated using an aqueous electrolyte, showed a high cycling stability with manufacturing methods that can be scaled for 


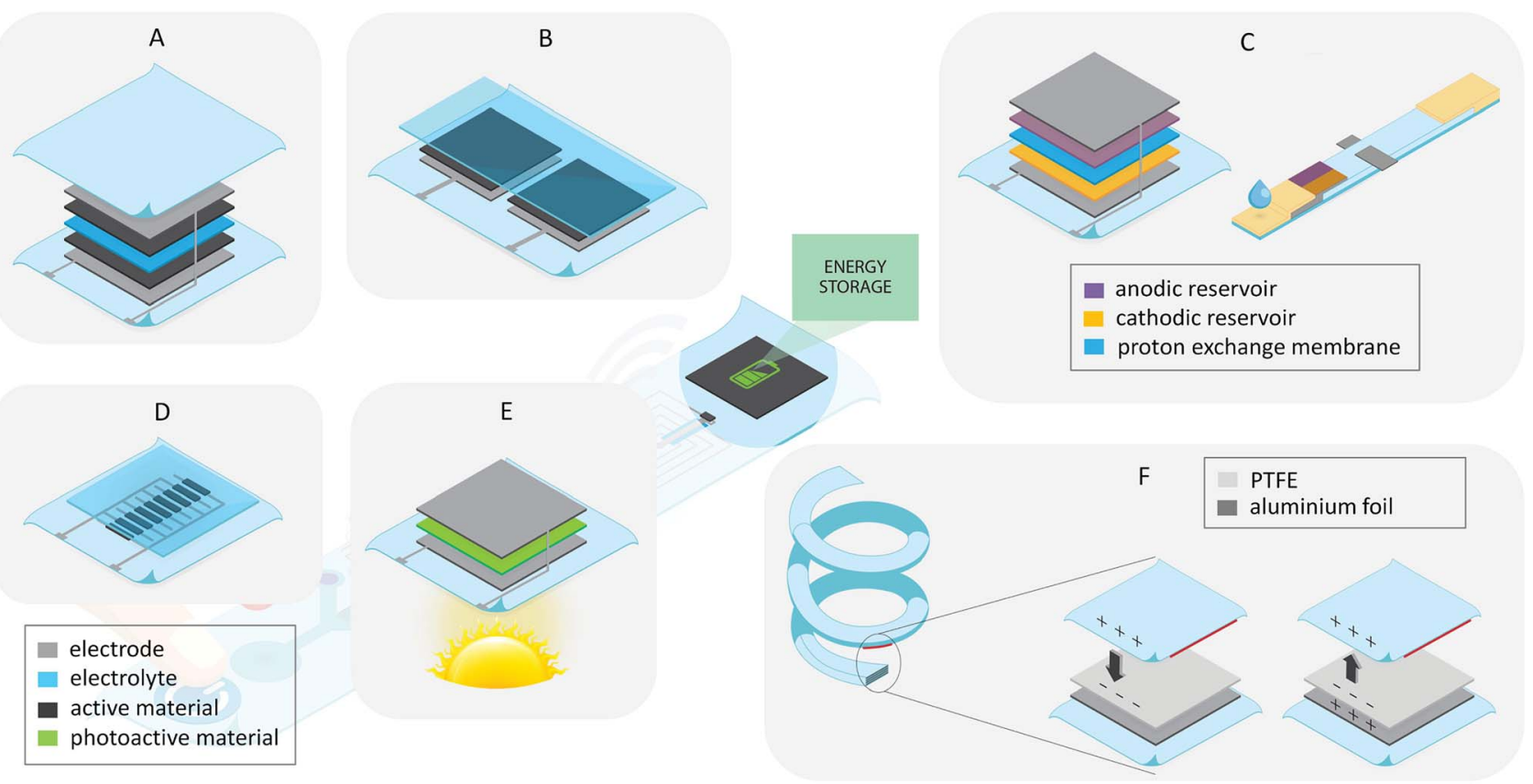

Fig. 10 Printed energy storage modules. Batteries can be either in stacked or parallel formats. (A) In a stacked configuration, a paper-based separator soaked with electrolyte is sandwiched between an anode and cathode, each printed on to a current collector on a paper substrate. (B) In a parallel format, two current collectors are printed side by side on a paper substrate, one with an anode material printed on top and one with cathode material. An electrolyte layer is printed or applied over these to complete the battery. (C) Biofuel cells can also utilize a stacked configuration with two electrodes on the outside and anodic and cathodic reservoirs on either side of a proton exchange membrane. Biofuel cells can also be implemented in a lateral flow format, where fuel (anolyte and catholyte) are spotted on to the test and electrodes make contact, completing the cell when water is added to the test strip. (D) Supercapacitors can have either a stacked format - similar to batteries - or an interdigitated configuration with active material over the electrodes and a printed electrolyte layer covering this. (E) Solar cells have a photoactive material between a cathode and anode, printed on to paper or a transparent electrode through which sunlight is absorbed to generate power. (F) Nanogenerators utilize stretching or twisting of paper structures to generate energy. As an example, layers of polytetrafluoroethylene (PTFE) and aluminium foil on paper can be used to generate energy through contact and release of the paper surface to the PTFE surface.

Table 1 SWOT analysis of augmented paper-based diagnostics towards fulfilling the ASSURED criteria

\begin{tabular}{ll}
\hline Strengths & Opportunities
\end{tabular}

- Paper is easily available

- Paper is low-cost

- Paper is accepted as a reliable rapid test format

- Paper is well-suited to unit operations towards ASSURED

- Devices are equipment free

- Laboratory facilities are not required

- Minimal training required

- Passive fluid flow

- Enables automated readout

- Enables data collection

- On-board power

- Maintenance free
- Easy distribution of devices to remote sites

- Rapidly growing paper-based diagnostics market

- Rapidly growing printed electronics and wearables markets

- Test multiplexing

- Enables decentralized testing

- Manufacturing on a distributed basis

- Scalable manufacturing processes

- Environmentally friendly

- Many unexplored technical components

- Tracking and containing disease outbreaks

- Addresses unique challenges of under-resourced settings
- Technological hurdles

- Seamless integration of components

- Robust and integrated hybrid electronics

- Speed and reliability of printed electronics

- Fluidic control and reagent lifetimes

- Fully quantitative results
- Conventional and standard commercial devices

- Batch to batch consistency

- Shelf-life of devices

- Logistics and environmental impact

- Regulatory approvals

- Market and user acceptance

- Data security and certification 
mass production. ${ }^{\mathbf{1 8 0}}$ Applications for wearables or portable devices are suggested, and could be extended to paper-based ASSURED devices for energy storage modules to power onboard components required for actuation, detection and display.

\subsection{Solar cells}

Solar photovoltaic systems generate direct current electricity from the sun's rays. Recent developments based on polymers focus on thin-film inorganic, organic and dye-sensitized solar cell technologies, ${ }^{27}$ which can generally be printed using roll-toroll techniques in custom shapes and sizes. Dye-sensitized solar cells on paper substrates ${ }^{\mathbf{1 8 1}}$ and printed photovoltaic cells on paper substrates that are recyclable ${ }^{92}$ have been investigated, as well as solar cells inserted in to textiles using conventional weaving processes. ${ }^{\mathbf{1 8 2}}$ Novel papers with a balance between transparency and light scattering have been developed for realizing effective paper-based solar cells. ${ }^{183}$ Oxidative chemical vapour deposition has been implemented to produce solar cells on newspaper and standard printing paper, with a typical structure consisting of an anode and a cathode with a photoactive layer $^{\mathbf{1 8 4}}$ (Fig. 10E). Polymer solar cells with semitransparent top electrodes ${ }^{\mathbf{1 8 5}}$ and cellulose-based electrodes implemented for dye-sensitized solar cells ${ }^{186}$ have demonstrated high efficiency organic photovoltaics despite the roughness of the paper substrates.

The latter paper-based dye-sensitized solar cells demonstrated compact, robust devices which could be sufficient for powering of components that form part of ASSURED paperbased diagnostic devices. Multiple cells would be required to obtain voltages and currents high enough to drive LEDs, for example, but could be a feasible, durable and environmentally sustainable solution for on-board power for ASSURED devices.

\subsection{Nanogenerators}

Nanogenerators convert thermal or mechanical energy produced by small-scale physical changes into electricity. Thermoelectric generators (TEGs) convert small changes in temperature to electric voltage, and are often utilized in wearable technologies. ${ }^{187}$ A recent review of fiber-based TEGs summarizes the materials, operating principles and device structures, as well as example applications. ${ }^{\mathbf{1 8 8}}$ Spray coating and screen printing are commonly used to realize printed functionality in textile-based TEG devices. Paper-based TEGs have been developed and, as an example, utilize waste heat and folding or origami techniques to generate energy. ${ }^{189}$ Stacking or folding of layers enables higher power densities to be achieved while maintaining device flexibility.

Organic TEGs have been developed by Otego, ${ }^{190}$ utilizing printable, low-cost semiconductors which can be massproduced using roll-to-roll techniques and then folded using automated, large-scale processes into small cubes to create a compact power source. TEG devices could be applicable for powering components such as wireless communication or sensing modules for ASSURED point-of-care diagnostics in resource-limited settings, where the environment is harsh and temperature fluctuations are extreme.

Other examples of nanogenerators are those that are capable of harvesting energy from human movements, and can be implemented on paper using twistable and stretchable designs (Fig. 10F), making them well-suited to paper-based diagnostics. Origami-based nanogenerators and self-powered pressure sensors have been showcased, ${ }^{191}$ as well as paper-based nanogenerators for power generation and active sensor implementation ${ }^{192}$ in which the action of turning page of a book can generate currents and voltages sufficient to power LEDs, for example.

\section{Integration}

Integration of the various printed functionality components summarized in this work is required for fully ASSURED paperbased point-of-care diagnostics to be realized. Readout of lateral flow devices has evolved from using manual visual analysis to instrumented and automated readout, ${ }^{193}$ but for fully integrated paper-based diagnostics with printed functionality, microfluidics and electronics need to be combined on the same paper substrate. As each of these aspects is suited to different paper characteristics, ${ }^{\mathbf{1 9 4}, \mathbf{1 9 5}}$ substrate pre-treatment and modification, ${ }^{196}$ substrate integration, ${ }^{197}$ and various printing and patterning techniques to optimize both electronic and fluidic performance ${ }^{41,145,198-200}$ have been investigated.

Point-of-care diagnostic devices on flexible substrates ${ }^{\mathbf{1 0 2}}$ and integration of printed electronics in to the real world ${ }^{\mathbf{2 0 1}}$ have been explored with potential for future wearable applications. Printed diodes, antennas and electrochromic displays have been combined to form an all-printed electronic tag for IoT applications. ${ }^{202}$ Examples of integrated flexible devices include a connected electrochemical sensing platform resulting from a collaboration between Acreo $\mathrm{AB}$ and Linköping University, ${ }^{195,201,203}$ a device to track micronutrient deficiencies, ${ }^{204}$ and a disposable system to measure hydrogen peroxide and total cholesterol, which is compatible with a mobile application for read-out of results. ${ }^{205}$

Paper-based solutions of co-fabricated microfluidics, electronics and batteries ${ }^{32}$ and electrochromic readout for electrochemical sensing with on-board power ${ }^{111}$ have been showcased, as well as connected paper-based temperature ${ }^{206}$ and gas $^{207}$ sensors implemented on RFID wireless platforms. Paper-based piezoresistive pressure sensors combined with visual readout for medical applications such as measuring bandage compression, ${ }^{208}$ and infant birth weight ${ }^{209}$ have also been presented. Paper-based diagnostics that have been introduced to the market have been summarized ${ }^{2}$ and include developments by Diagnostics For All (DfA) ${ }^{\mathbf{2 1 0}}$ for liver function and nucleic acid testing, text-reported blood typing by haemokinesis, ${ }^{105}$ and lateral flow devices for infectious diseases by INSiGHT. ${ }^{211}$

Established techniques such as roll-to-roll printing can be utilized for mass production of printed electronics and functional systems. The pilot factory established by PrintoCent ${ }^{27}$ has assembly processes in place for production of hybrid electronic 
devices. Lateral flow and dipstick tests already use established mass manufacturing techniques, but for newly developed paper-based diagnostics, scalability of microfluidic structure patterning, deposition of assay components, and assembly of multi-layered devices will be crucial for successful mass production. ${ }^{2,199}$ Substrates, inks, manufacturing processes, assembly and integration as well as fabrication time are important cost factors to consider for the successful commercialization of paper-based diagnostics with enhanced functionality.

\section{Conclusion and outlook}

This work emphasizes the recent developments made towards integrated printed and paper-based diagnostic solutions, and the variety of functional components that have been and can potentially be realized to enhance functionality. Specifically, the review focusses on the functionalities developed to assist with meeting the ASSURED criteria, and shows that paper is wellsuited for the development of the unit operations needed to achieve this. Although headway has been made, seamless integration of the different building blocks into a functional solution, conceptualized by Fig. 2A, requires further development for truly ASSURED paper-based devices to be realized. This review has highlighted both the need and future opportunities to connect and integrate the components into functional systems. Table 1 provides a summary of the strengths, weaknesses, opportunities and threats (SWOT) of paper-based diagnostics with added functionalities towards fulfillment of the ASSURED criteria.

Fully integrated systems are crucial as they will remove subjective and often incorrect human interpretation of results and allow for robust data collection and data security. This would enable resource-limited settings to improve diagnosis and healthcare infrastructure in the long-term. Beyond ASSURED, additional functionalities are required for modern clinical practises and crisis readiness. Data acquisition and communication are important considerations which a systems approach can ensure through digitized data and connectivity. Rapid data collection is of increasing importance, especially with contagious diseases and outbreaks, and could be included as an overarching function in augmented ASSURED paper-based diagnostic solutions. However, data security remains a challenge where a lack of data certification and confidentiality can result in false diagnosis, stigma, and destruction of lives. This will be a crucial challenge to address as digitization of data evolves and information needs to be more tightly managed.

As the need for more data to be collected and processed grows, so will the need for robust, compact and interconnected paper-based electronics. Many current solutions make use of hybrid electronics, which have the speed of traditional electronics, but interconnects are not robust and the solution is not fully integrated. In contrast, flexible, paper-based electronics are often limited in terms of speed, and addressing these constraints will need to form part of the roadmap moving forward.
In addition to the technical integration challenges using printed functionalities are the unique challenges faced in under-resourced settings. These have been discussed in the Introduction and have been highlighted by case studies, ${ }^{212}$ and play a crucial role in the development of successful paper-based ASSURED solutions.

Successful implementation will also depend on the ability to scale manufacture to produce high volumes of devices, along with the supply chain infrastructure to ensure deployment to remote settings. From an environmental impact perspective, using paper as a platform may be beneficial as it can be considered $\mathrm{CO}_{2}$ neutral, i.e. incineration of paper does not unnecessarily produce more of a carbon burden. Incineration of paper also deals with the issue of sterility, where in contrast plastic-based medical devices are typically dumped and can pose risks to the public, particularly in resource-limited settings. However, if incineration of paper-based devices starts to occur on a large scale, the logistics and environmental impact of this will need to be evaluated. Self-igniting devices could be explored, as well as biodegradable substrates and materials, where the latter would require further investigations regarding shelf-life and robustness of the devices.

Only once the above mentioned barriers have been overcome will the real impact of these solutions be realizable. Ultimately, the success of paper-based diagnostics lies in the development and optimization of automation, ease-of-use, and shelf life, while incorporating feedback from users performing field tests in resource-limited settings. Scalability, deployment and disposability will need to form part of the development process from the early stages for large scale feasible, sustainable solutions to be realized. If this can be achieved, fully ASSURED paper-based diagnostics with augmented functionalities for data, connectivity and environmental sustainability for resource-limited settings are a viable solution for the future.

\section{Conflicts of interest}

There are no conflicts of interest to declare.

\section{Acknowledgements}

The authors gratefully acknowledge the Council for Scientific and Industrial Research (CSIR), South Africa and Karlsruhe Institute of Technology (KIT), Germany for funding this work. Special thanks are extended to Hildegard Prigge for providing the illustrations for this review.

\section{References}

1 D. Mabey, R. W. Peeling, A. Ustianowski and M. D. Perkins, Nat. Rev. Microbiol., 2004, 2, 231-240.

2 K. Yamada, H. Shibata, K. Suzuki and D. Citterio, Lab Chip, 2017, 17, 1206-1249.

3 A. K. Yetisen, M. S. Akram and C. R. Lowe, Lab Chip, 2013, 13, 2210-2251. 
4 J. S. Chang, A. F. Facchetti and R. Reuss, IEEE Journal on Emerging and Selected Topics in Circuits and Systems, 2017, 7, 7-26.

5 S. Khan, L. Lorenzelli and R. S. Dahiya, IEEE Sens. J., 2015, 15, 3164-3185.

6 F. Hoeng, A. Denneulin and J. Bras, Nanoscale, 2016, 8, 13131-13154.

7 J. Kim, R. Kumar, A. J. Bandodkar and J. Wang, Adv. Electron. Mater., 2016, 3, 1600260.

8 D. Liana, B. Raguse, J. Justin Gooding and E. Chow, Sensors, 2012, 12, 11505-11526.

9 N. A. Meredith, C. Quinn, D. M. Cate, T. H. Reilly, J. Volckens and C. S. Henry, Analyst, 2016, 141, 1874-1887.

10 D. Cate, J. Adkins, J. Mettakoonpitak and C. Henry, Anal. Chem., 2015, 87, 19-41.

11 J. Hu, S. Wang, L. Wang, F. Li, B. Pingguan-Murphy, T. Lu and F. Xu, Biosens. Bioelectron., 2014, 54, 585-597.

12 A. Martinez, S. Phillips, E. Carrilho, S. Thomas III, H. Sindi and G. Whitesides, Anal. Chem., 2008, 80, 3699-3707.

13 J. Delaney, E. Doeven, A. Harsant and C. Hogan, Anal. Chim. Acta, 2013, 803, 123-127.

14 P. Bezuidenhout, S. Smith, K. Land and T. H. Joubert, 2017 IEEE AFRICON, 2017, pp. 83-87.

15 B. Liu, D. Du, X. Hua, X.-Y. Yu and Y. Lin, Electroanalysis, 2014, 26, 1214-1223.

16 C. Zhao, M. M. Thuo and X. Liu, Sci. Technol. Adv. Mater., 2013, 14, 054402.

17 N. Engel, M. Davids, N. Blankvoort, N. Pai, K. Dheda and M. Pai, Trop. Med. Int. Health, 2015, 20, 493-500.

18 W. D. Meyer, PathCare Pathology Forum - Point of Care, Vol 4 No 2, Dietrich Voight Mia Partners, Goodwood, 1st edn, 2013, ch. 7, pp. $24-25$.

19 G. A. Posthuma-Trumpie, J. Korf and A. van Amerongen, Anal. Bioanal. Chem., 2009, 393, 569-582.

20 A. W. Martinez, S. T. Phillips, Z. Nie, C.-M. Cheng, E. Carrilho, B. J. Wiley and G. M. Whitesides, Lab Chip, 2010, 10, 2499-2504.

21 Y. Xia, J. Si and Z. Li, Biosens. Bioelectron., 2016, 77, 774789.

22 M. Sher, R. Zhuang, U. Demirci and W. Asghar, Expert Rev. Mol. Diagn., 2017, 17, 351-366.

23 A. Nilghaz, L. Guan, W. Tan and W. Shen, ACS Sens., 2016, 1, 1382-1393.

24 E. Maxwell, A. Mazzeo and G. Whitesides, MRS Bull., 2013, 38, 309-314.

25 G. Zheng, Y. Cui, E. Karabulut, L. Wågberg, H. Zhu and L. Hu, MRS Bull., 2013, 38, 320-325.

26 T. Liang, X. Zou and A. Mazzeo, Proc. SPIE, 2016, 9836, 98361D.

27 T. Piila, A. Juusti, M. Kylmänen and K. Ikonen, Printed electronics and diagnostic products: PrintoCent designer's handbook, Neficon Finland Oy, 2015.

28 G. Grau, J. Cen, H. Kang, R. Kitsomboonloha, W. J. Scheideler and V. Subramanian, Flexible Printed Electron., 2016, 1, 023002.

29 H. Wu, S. W. Chiang, W. Lin, C. Yang, Z. Li, J. Liu, X. Cui, F. Kang and C. P. Wong, Sci. Rep., 2014, 4, 6275.
30 H. A. Andersson, A. Manuilskiy, S. Haller, M. Hummelgard, J. Sidén, C. Hummelgard, H. Olin and H.-E. Nilsson, Nanotechnology, 2014, 25, 094002.

31 A. Siegel, S. Phillips, M. Dickey, N. Lu, Z. Suo and G. Whitesides, Adv. Funct. Mater., 2010, 20, 28-35.

32 M. M. Hamedi, V. E. Campbell, P. Rothemund, F. Güder, D. C. Christodouleas, J.-F. Bloch and G. M. Whitesides, Adv. Funct. Mater., 2016, 26, 2446-2453.

33 A. Ostfeld, I. Deckman, A. Gaikwad, C. Lochner and A. Arias, Sci. Rep., 2015, 5, 15959.

34 J. Chang, X. Zhang, T. Ge and J. Zhou, Org. Electron., 2014, 15, 701-710.

35 Y. Gao, H. Li and J. Liu, PLoS One, 2013, 8, 1-8.

36 T. Meiss, R. Wertschützky and B. Stoeber, 2014 IEEE 27th Int. Conf., MEMS, 2014, pp. 536-539.

37 X. Zhang, T. Ge and J. S. Chang, Org. Electron., 2015, 26, 371-379.

38 E. Sowade, E. Ramon, K. Mitra, C. Martinez-Domingo, M. Pedro, J. Pallares, F. Loffredo, F. Villani, H. Gomes, L. Teres and R. Baumann, Sci. Rep., 2016, 6, 33490.

39 A. D. M. Tongfen Liang and X. Zou, Proc. SPIE, 2016, 9836, 14.

40 Y.-J. Kwon, Y. D. Park and W. H. Lee, Materials, 2016, 9, 650. 41 G. Jenkins, Y. Wang, Y. Xie, Q. Wu, W. Huang, L. Wang and X. Yang, Microfluid. Nanofluid., 2015, 19, 251-261.

42 J. Huang, H. Zhu, Y. Chen, C. Preston, K. Rohrbach, J. Cumings and L. Hu, ACS Nano, 2013, 7, 2106-2113.

43 A. S. Dahiya, C. Opoku, G. Poulin-Vittrant, N. Camara, C. Daumont, E. G. Barbagiovanni, G. Franzo, S. Mirabella and D. Alquier, ACS Appl. Mater. Interfaces, 2017, 9, 573-584.

44 K. Y. Mitra, M. Polomoshnov, C. Martinez Domingo, D. Mitra, E. Ramon and R. Baumann, Adv. Electron. Mater., 2017, 3, 1770053.

45 E. Bihar, Y. Deng, T. Miyake, M. Saadaoui, G. G. Malliaras and M. Rolandi, Sci. Rep., 2016, 6, 27582.

46 I. Gualandi, M. Marzocchi, A. Achilli, D. Cavedale, A. Bonfiglio and B. Fraboni, Sci. Rep., 2016, 6, 33637.

47 Acreo, https:/www.acreo.se/expertise/printed-electronictechnologies, 2016.

48 Y. Lin, D. Gritsenko, Q. Liu, X. Lu and J. Xu, ACS Appl. Mater. Interfaces, 2016, 8, 20501-20515.

49 R. Courtland, IEEE Spectrum, 2017, 54, 7-8.

50 J. C. Cunningham, P. R. DeGregory and R. M. Crooks, Annu. Rev. Anal. Chem., 2016, 9, 183-202.

51 D. Sritharan and E. Smela, Polymers, 2016, 8, 400.

52 J. Kim, S. Yun, S. K. Mahadeva, K. Yun, S. Y. Yang and M. Maniruzzaman, Sensors, 2010, 10, 1473-1485.

53 M. Amjadi and M. Sitti, ACS Nano, 2016, 10, 10202-10210.

54 S. K. Mahadeva, K. Walus and B. Stoeber, ACS Appl. Mater. Interfaces, 2014, 6, 7547-7553.

55 S. Emamian, B. B. Narakathu, A. A. Chlaihawi and M. Z. Atashbar, Procedia Eng., 2016, 168, 688-691.

56 S. K. Mahadeva, K. Walus and B. Stoeber, 2015, 28th IEEE International Conference on Micro Electro Mechanical Systems, MEMS, 2015, pp. 861-864.

57 E. Lemaire, R. Moser, C. Borsa, H. Shea and D. Briand, Procedia Eng., 2015, 120, 360-363. 
58 Y. Matsuda, S. Shibayama, K. Uete, H. Yamaguchi and T. Niimi, Anal. Chem., 2015, 87, 5762-5765.

59 E. A. Phillips, R. Shen, S. Zhao and J. C. Linnes, Lab Chip, 2016, 16, 4230-4236.

60 T. Fischer, N. Wetzold, H. Elsner, L. Kroll and A. C. Hübler, Nanomater. Nanotechnol., 2011, 1, 18-23.

61 G. Mattana and D. Briand, Mater. Today, 2016, 19, 88-99.

62 L. Gih-Keong and M. Shrestha, Micromachines, 2017, 8, 119.

63 J. M. Nassar, M. D. Cordero, A. T. Kutbee, M. A. Karimi, G. A. T. Sevilla, A. M. Hussain, A. Shamim and M. M. Hussain, Adv. Mater. Technol., 2016, 1, 1600004.

64 C. M. Silveira, T. Monteiro and M. G. Almeida, Biosensors, 2016, 6(51), 1-17.

65 J. Mettakoonpitak, K. Boehle, S. Nantaphol, P. Teengam, J. A. Adkins, M. Srisa-Art and C. S. Henry, Electroanalysis, 2016, 28, 1420-1436.

66 M. Zhang, A. Halder, X. Cao, C. Hou and Q. Chi, GraphenePaper Based Electrochemical Sensors, in Electrochemical Sensors Technologies, INTECH, 2017, ch. 3, pp. 33-62.

67 S. Smith, P. Bezuidenhout, M. B. Mbanjwa, H. Zheng, M. Conning, N. Palaniyandy, K. Ozoemena and K. Land, Proc. SPIE, 2017, 10036.

68 G. Martines, A. Tavares, M. Fortunato and E. amd Sales, Sci. Rep., 2017, 7, 1-10.

69 V. B. C. Lee, N. F. Mohd-Naim, E. Tamiya and M. U. Ahmed, Anal. Sci., 2018, 34, 7-18.

70 P. Bezuidenhout, S. Smith and T.-H. Joubert, Appl. Sci., 2018, 8, 968.

71 K. Koren and M. Kühl, Sens. Actuators, B, 2015, 210, 124128.

72 PST Sensors, https://www.pstsensors.com/, 2017.

73 M. Mraovic, T. Muck, M. Pivar, J. Trontelj and A. Pletersek, Sensors, 2014, 14, 13628-13643.

74 F. Guder, A. Ainla, J. Redston, B. Mosadegh, A. Glavan, T. J. Martin and G. M. Whitesides, Angew. Chem., Int. Ed., 2016, 55, 5727-5732.

75 D. Barmpakos, A. Segkos, C. Tsamis and G. Kaltsas, J. Phys.: Conf. Ser., 2017, 931, 012003.

76 L. Huang, P. Jiang, D. Wang, Y. Luo, M. Li, H. Lee and R. A. Gerhardt, Sens. Actuators, B, 2014, 197, 308-313.

77 A. Quddious, S. Yang, M. M. Khan, F. A. Tahir, A. Shamim, K. N. Salama and H. M. Cheema, Sensors, 2016, 16, 2073.

78 T.-L. Ren, H. Tian, D. Xie and Y. Yang, Sensors, 2012, 12, 6685-6694.

79 Y. Kawahara, S. Hodges, N.-W. Gong, S. Olberding and J. Steimle, IEEE Pervasive Comput., 2014, 30-38.

80 S. Gong, W. Schwalb, Y. Wang, Y. Chen, Y. Tang, K. Si, B. Shirinzadeh and W. Cheng, Nat. Commun., 2014, 5, 1-8.

81 M. Jung, K. Kim, B. Kim, H. Cheong, K. Shin, O.-S. Kwon, J.-J. Park and S. Jeon, ACS Appl. Mater. Interfaces, 2017, 9, 26974-26982.

82 G. Pace, A. Grimoldi, M. Sampietro, D. Natali and M. Caironi, Semicond. Sci. Technol., 2015, 30, 104006.

83 C. Xie and F. Yan, Small, 2017, 13, 1701822.

84 R. S. Aga, J. P. Lombardi, C. M. Bartsch and E. M. Heckman, IEEE Photon. Technol. Lett., 2014, 26, 305-308.
85 S. Lilliu, M. Böberl, M. Sramek, S. F. Tedde, J. E. Macdonald and O. Hayden, Thin Solid Films, 2011, 520, 610-615.

86 A. Pierre, I. Deckman, P. B. Lechene and A. C. Arias, Adv. Mater., 2015, 27, 6411-6417.

87 S. Jacob, M. Benwadih, J. Bablet, M. Charbonneau, A. Aliane, A. Plihon and A. Revaux, Proc. IEEE IEDM, 2015, pp. 19.5.1-19.5.4.

88 L. Zhang, T. Wu, Y. Guo, Y. Zhao, X. Sun, Y. Wen, G. Yu and Y. Liu, Sci. Rep., 2013, 3, 1080.

89 ISORG, https://www.isorg.fr/, 2017.

90 IDTechEx Research, https:/www.idtechex.com, 2016.

91 P. T. Gomathi, P. Sahatiya and S. Badhulika, Adv. Funct. Mater., 2017, 27, 1701611.

92 A. Hübler, B. Trnovec, T. Zillger, M. Ali, N. Wetzold, M. Mingebach, A. Wagenpfahl, C. Deibel and V. Dyakonov, Adv. Energy Mater., 2011, 1, 1018-1022.

93 A. Sou, S. Jung, E. Gili, V. Pecunia, J. Joimel, G. Fichet and H. Sirringhaus, Org. Electron., 2014, 15, 3111-3119.

94 Q. Cao, H.-S. Kim, N. Pimparkar, J. Kulkarni, C. Wang, M. Shim, K. Roy, M. Alam and J. Rogers, Nature, 2008, 454, 495-500.

95 K. Myny, S. Smout, M. Rockele, A. Bhoolokam, T. Ke, S. Steudel, B. Cobb, A. Gulati, F. Rodriguez, K. Obata, M. Marinkovic, D.-V. Pham, A. Hoppe, G. Gelinck, J. Genoe, W. Dehaene and P. Heremans, Sci. Rep., 2014, 4, 7398.

96 S. Mandal and Y.-Y. Noh, Semicond. Sci. Technol., 2015, 30, 064003.

97 P. Vilmi, M. Nelo, J.-V. Voutilainen, J. Palosaari, J. Pörhönen, S. Tuukkanen, H. Jantunen, J. Juuti and T. Fabritius, Flexible Printed Electron., 2016, 1, 025002.

98 VTT Technical Research Centre of Finland Ltd., https:// www.vttresearch.com/, 2017.

99 M. Rehman, G. Siddiqui, J. Gul, S.-W. Kim, J. Hwan Lim and K. Choi, Sci. Rep., 2016, 6, 36195.

100 D. H. Lien, Z. K. Kuo, T. H. Huang, Y. C. Liao, S. C. Lee and H. He, Symposium on VLSI Technology, 2014, pp. 1-2.

101 B.-H. Lee, D.-I. Lee, H. Bae, H. Seong, S.-B. Jeon, M.-L. Seol, J.-W. Han, M. Meyyappan, S.-G. Im and Y.-K. Choi, Sci. Rep., 2016, 6, 38389.

102 S. Wang, T. Chinnasamy, M. A. Lifson, F. Inci and U. Demirci, Trends Biotechnol., 2016, 34, 909-921.

103 R. S. Deol, H. W. Choi, M. Singh and G. E. Jabbour, IEEE Sens. J., 2015, 15, 3186-3195.

104 M. R. Fernández, E. Z. Casanova and I. G. Alonso, Sustainability, 2015, 7, 10854.

105 Haemokinesis, https:/www.haemokinesis.com/products/ bio-active-paper-tests/glif-bg, 2018.

106 A. C. Siegel, S. T. Phillips, B. J. Wiley and G. M. Whitesides, Lab Chip, 2009, 9, 2775-2781.

107 H. Shin, B. Yoon, I. S. Park and J.-M. Kim, Nanotechnology, 2014, 25, 094011.

108 J. Kawahara, P. Andersson Ersman, D. Nilsson, K. Katoh, Y. Nakata, M. Sandberg, M. Nilsson, G. Gustafsson and M. Berggren, J. Polym. Sci., Part B: Polym. Phys., 2013, 51, 265-271. 
109 P. A. Ersman, J. Kawahara and M. Berggren, Org. Electron., 2013, 14, 3371-3378.

110 P. Andersson, D. Nilsson, P.-O. Svensson, M. Chen, A. Malmstrom, T. Remonen, T. Kugler and M. Berggren, Adv. Mater., 2002, 14, 1460-1464.

111 H. Liu and R. Crooks, Anal. Chem., 2012, 84, 2528-2532.

112 D. D. Liana, B. Raguse, J. J. Gooding and E. Chow, ACS Appl. Mater. Interfaces, 2015, 7, 19201-19209.

113 S. Meier, D. Tordera, A. Pertegas, C. Carmona, E. Orti and H. Bolink, Mater. Today, 2014, 17, 217-223.

114 A. Asadpoordarvish, A. Sandstrom, C. Larsen, R. Bollstrom, M. Toivakka, R. Osterbacka and L. Edman, Adv. Funct. Mater., 2015, 25, 3238-3245.

115 T. Lanz, A. Sandstrom, S. Tang, P. Chabrecek, U. Sonderegger and L. Edman, Flexible Printed Electron., 2016, 1, 025004.

116 J. Liu, J. Oliva, K. Tong, F. Zhao, D. Chen and Q. Pei, Sci. Rep., 2017, 7, 1524.

117 J. Zimmermann, L. Porcarelli, T. Rodlmeier, A. SanchezSanchez, D. Mecerreyes and G. Hernandez-Sosa, Adv. Funct. Mater., 2018, 28, 1705795.

118 J. Y. Kim, S. H. Park, T. Jeong, M. J. Bae, S. Song, J. Lee, I. T. Han, D. Jung and S. Yu, IEEE Trans. Electron Devices, 2010, 57, 1470-1474.

119 W. Gao, M. Saqib, L. Qi, W. Zhang and G. Xu, Curr. Opin. Electrochem., 2017, 3, 4-10.

120 E. M. Gross, H. E. Durant, K. N. Hipp and R. Y. Lai, ChemElectroChem, 2017, 4, 1594-1603.

121 D.-Y. Yoon, T.-Y. Kim and D.-G. Moon, Curr. Appl. Phys., 2010, 10, e135-e138.

122 S. Purandare, E. Gomez and A. Steckl, Nanotechnology, 2014, 25, 094012.

123 S. J. Telfer and M. D. McCreary, SID Symposium Dig. Tech. Pap., 2016, vol. 47, pp. 574-577.

124 D. Y. Kim and A. J. Steckl, ACS Appl. Mater. Interfaces, 2010, 2, 3318-3323.

125 P. Bai, R. Hayes, M. Jin, L. Shui, Z. Yi, L. Wang, X. Zhang and G. Zhou, Prog. Electromagn. Res., 2014, 147, 95-116.

126 intelliPaper, https://www.intellipaper.info/, 2017.

127 S. Madakam, R. Ramaswamy and S. Tripathi, Journal of Computer and Communications, 2015, 3, 164-173.

128 NXP Semiconductors, https://www.nxp.com/products/ wireless-connectivity:WIRELESS-CONNECTIVITY, 2017.

129 E. Ghafar-Zadeh, Sensors, 2015, 15, 3236-3261.

130 S. Chia, A. Zalzala, L. Zalzala and A. Karim, IEEE Technol. Soc. Mag., 2013, 32, 36-43.

131 B. Chowdhury and R. Khosla, ICIS 2007, 2007, pp. 363-368.

132 F. Rahman, M. Z. A. Bhuiyan and S. I. Ahamed, Future Gener. Comput. Syst., 2016, 72, 339-352.

133 L. Yang and M. Tentzeris, IEEE MTT-S Int. Microwave Symposium Digest, 2007, pp. 1633-1636.

134 M. Kisic, B. Dakic, M. Damnjanovic, A. Menicanin, N. Blaz and L. Zivanov, IEEE ISSE, 2013, pp. 263-267.

$135 \mathrm{H}$. Abutarboush and A. Shamim, IEEE Antennas Wirel. Propag. Lett., 2012, 11, 1234-1237.

136 G. Shaker, A. Rida, S. Safavi-Naeini, M. Tentzeris and S. Nikolaou, Proc. EUCAP 2011, 2011, pp. 3001-3004.
137 L. Yang, A. Rida, R. Vyas and M. Tentzeris, IEEE Trans. Microwave Theory Tech., 2007, 55, 2894-2901.

138 E. Sipilä, J. Virkki, J. Wang, L. Sydänheimo and L. Ukkonen, Int J. Antennas Propag., 2016, 2016, 3694198.

139 H. He, L. Sydänheimo, J. Virkki and L. Ukkonen, Int J. Antennas Propag., 2016, 2016, 9265159.

140 T. Çiftçi, B. Karaosmanoğlu and O. Ergül, IOP Conf. Ser. Mater. Sci., 2016, vol. 120, p. 012005.

141 U. Kavcic, M. Maek and T. Muck, J. Imaging Sci. Technol., 2015, 59, 050504.

142 U. Kavcic, M. Pivar, M. Dokic, D. Gregor-Svetec, L. Pavlovic and T. Muck, Mater. Technol., 2014, 48, 261-267.

143 S. Smith, P. Bezuidenhout, K. Land, J. Korvink and D. Mager, RAPDASA 2017, 2017, pp. 243-249.

144 B. Cook, J. Cooper and M. Tentzeris, IEEE Trans. Microwave Theory Tech., 2013, 61, 4714-4723.

145 W. Su, B. S. Cook, Y. Fang and M. Tentzeris, Sci. Rep., 2016, 6, 35111.

146 J. Zhang, G. Y. Tian, A. M. J. Marindra, A. I. Sunny and A. B. Zhao, Sensors, 2017, 17(265), 1-33.

147 F. Alimenti, C. Mariotti, V. Palazzi, M. Virili, G. Orecchini, P. Mezzanotte and L. Roselli, J. Low Power Electron. Appl., 2015, 5, 151-164.

148 M. Yuan, E. C. Alocilja and S. Chakrabartty, IEEE Trans. Biomed. Circuits Syst., 2016, 10, 799-806.

149 Arjowiggins Powercoat, https://powercoatpaper.com/ products/powercoat-alive/, 2017.

150 T. Nguyen, A. Fraiwan and S. Choi, Biosens. Bioelectron., 2014, 54, 640-649.

151 A. M. Gaikwad, A. C. Arias and D. A. Steingart, Energy Technol., 2015, 3, 305-328.

152 L. Nyholm, G. Nystrom, A. Mihranyan and M. Stromme, Adv. Mater., 2011, 23, 3751-3769.

153 B. Yao, J. Zhang, T. Kou, Y. Song, T. Liu and Y. Li, Adv. Sci., 2017, 4, 1700107.

154 S. Pan, J. Ren, X. Fang and H. Peng, Adv. Energy Mater., 2016, 6, 1501867.

155 N. Thom, K. Yeung, M. Pillion and S. Phillips, Lab Chip, 2012, 12, 1768-1770.

156 S.-S. Chen, C.-W. Hu, I.-F. Yu, Y.-C. Liao and J.-T. Yang, Lab Chip, 2014, 14, 2124-2130.

157 A. Willert and R. R. Baumann, ISCDG 2013, 2013, pp. 1-4. 158 Z. Tehrani, T. Korochkina, S. Govindarajan, D. Thomas, J. O'Mahony, J. Kettle, T. Claypole and D. Gethin, Org. Electron., 2015, 26, 386-394.

159 E. Lee and J. wook amd Kjeang, Biomicrofluidics, 2010, 4, 041301.

$160 \mathrm{H}$. Qu, J. Hou, Y. Tang, O. Semenikhin and M. Skorobogatiy, Can. J. Chem., 2017, 95, 169-173.

161 L. Hu, J. W. Choi, Y. Yang, S. Jeong, F. La Mantia, L.-F. Cui and Y. Cui, Proc. Natl. Acad. Sci. U.S.A., 2009, 106, 2149021494.

162 Q. Cheng, Z. Song, T. Ma, B. B. Smith, R. Tang, H. Yu, H. Jiang and C. K. Chan, Nano Lett., 2013, 13, 4969-4974.

163 A. Avoundjian, V. Galvan and F. A. Gomez, Micromachines, 2017, 8, 222. 
164 W. Gellett, M. Kesmez, J. Schumacher, N. Akers and S. Minteer, Electroanalysis, 2010, 22, 727-731.

165 C. Gonzalez-Solino and M. D. Lorenzo, Biosensors, 2018, 8, 11.

166 J. P. Esquivel, F. J. Del Campo, J. L. Gomez de la Fuente, S. Rojas and N. Sabate, Energy Environ. Sci., 2014, 7, 17441749.

167 H. Lee and S. Choi, Nano Energy, 2015, 15, 549-557.

168 A. Fraiwan, C. Dai, T. H. Nguyen and S. Choi, IEEE NEMS 2014, 2014, pp. 394-397.

169 A. Fraiwan, S. Mukherjee, S. Sundermier, H.-S. Lee and S. Choi, Biosens. Bioelectron., 2013, 49, 410-414.

170 A. Fraiwan and S. Choi, Biosens. Bioelectron., 2016, 83, 2732.

171 I. Shitanda, M. Momiyama, N. Watanabe, T. Tanaka, S. Tsujimura, Y. Hoshi and M. Itagaki, ChemElectroChem, 2017, 4, 2460-2463.

172 C. Narvaez Villarrubia, C. Lau, G. P. M. K. Ciniciato, S. Garcia, S. S. Sibbett, D. Petsev, S. Babanova, G. Gupta and P. Atanassov, Electronchemistry Communications, 2014, 45, 44-47.

173 Y. Wang, Y. Song and Y. Xia, Chem. Soc. Rev., 2016, 45, 5925-5950.

174 Y. J. Kang, H. Chung, C.-H. Han and W. Kim, Nanotechnology, 2012, 23, 065401.

175 L. Yuan, X. Xiao, T. Ding, J. Zhong, X. Zhang, Y. Shen, B. Hu, Y. Huang, J. Zhou and Z. Wang, Angew. Chem., Int. Ed., 2012, 51, 4934-4938.

176 B. Yao, L. Yuan, X. Xiao, J. Zhang, Y. Qi, J. Zhou, J. Zhou, B. Hu and W. Chen, Nano Energy, 2013, 2, 1071-1078.

177 M. P. Down, C. W. Foster, X. Ji and C. E. Banks, RSC Adv., 2016, 6, 81130-81141.

178 K.-H. Choi, J. Yoo, C. K. Lee and S.-Y. Lee, Energy Environ. Sci., 2016, 9, 2812-2821.

179 L. Ge, P. Wang, S. Ge, N. Li, J. Yu, M. Yan and J. Huang, Anal. Chem., 2013, 85, 3961-3970.

180 L. Shi, Y. Wang, W. Lai, Z. Jiang, R. Wang and C. Yang, 2017 18th International Conference on Electronic Packaging Technology, ICEPT, 2017, pp. 1277-1281.

181 B. Wang and L. L. Kerr, Sol. Energy Mater. Sol. Cells, 2011, 95, 2531-2535.

182 M. J. Yun, S. I. Cha, S. H. Seo, H. S. Kim and D. Y. Lee, Sci. Rep., 2015, 5, 11022.

183 Z. Fang, H. Zhu, Y. Yuan, D. Ha, S. Zhu, C. Preston, Q. Chen, Y. Li, X. Han, S. Lee, G. Chen, T. Li, J. Munday, J. Huang and L. Hu, Nano Lett., 2014, 14, 765-773.

184 N. W. Stauffer, Solar cells printed on paper, MIT Energy Initiative, 2011, https://energy.mit.edu/news/solar-cellsprinted-on-paper/.

185 L. Leonat, M. S. White, E. D. Glowacki, M. C. Scharber, T. Zillger, J. Ruhling, A. Hubler and N. S. Sariciftci, J. Phys. Chem. C, 2014, 118, 16813-16817.

186 F. Bella, D. Pugliese, L. Zolin and C. Gerbaldi, Electrochim. Acta, 2017, 237, 87-93.

187 A. R. M. Siddique, S. Mahmud and B. V. Heyst, Renewable Sustainable Energy Rev., 2017, 73, 730-744.
188 L. Zhang, S. Lin, T. Hua, B. Huang, S. Liu and X. Tao, Adv. Energy Mater., 2018, 8, 1700524.

189 J. P. Rojas, D. Conchouso, A. Arevalo, D. Singh, I. G. Foulds and M. M. Hussain, Nano Energy, 2017, 31, 296-301.

190 Otego, https://www.otego.de/en/, 2018.

191 P.-K. Yang, Z.-H. Lin, K. C. Pradel, L. Lin, X. Li, X. Wen, J.-H. He and Z. L. Wang, ACS Nano, 2015, 9, 901-907.

192 Q. Zhong, J. Zhong, B. Hu, Q. Hu, J. Zhou and Z. L. Wang, Energy Environ. Sci., 2013, 6, 1779-1784.

193 W. C. Mak, V. Beni and A. P. Turner, Trends Anal. Chem., 2016, 79, 297-305.

194 M. M. Hamedi, A. Ainla, F. Güder, D. C. Christodouleas, M. T. Fernández-Abedul and G. M. Whitesides, Adv. Mater., 2016, 28, 5054-5063.

195 V. Beni, D. Nilsson, P. Arven, P. Norberg, G. Gustafsson and a. P. F. Turner, ECS J. Solid State Sci. Technol., 2015, 4, S3001-S3005.

196 Y. Wang, H. Guo, J. Chen, E. Sowade, Y. Wang, K. Liang, K. Marcus, R. R. Baumann and Z. sheng Feng, ACS Appl. Mater. Interfaces, 2016, 8, 26112-26118.

197 H. Shafiee, W. Asghar, F. Inci, M. Yuksekkaya, M. Jahangir, M. Zhang, N. Durmus, U. Gurkan, D. Kuritzkes and U. Demirci, Sci. Rep., 2015, 5, 8719.

198 D. R. Ballerini, X. Li and W. Shen, Microfluid. Nanofluid., 2012, 13, 769-787.

199 C. R. Mace and R. N. Deraney, Microfluid. Nanofluid., 2014, 16, 801-809.

200 X. Jiang and Z. Fan, Annu. Rev. Anal. Chem., 2016, 9, 203222.

201 M. Berggren, D. T. Simon, D. Nilsson, P. Dyreklev, P. Norberg, S. Nordlinder, P. A. Ersman, G. Gustafsson, J. J. Wikner, J. Hederen and H. Hentzell, Adv. Mater., 2016, 28, 1911-1916.

202 N. Sani, M. Robertsson, P. Cooper, X. Wang, M. Svensson, P. Andersson Ersman, P. Norberg, M. Nilsson, D. Nilsson, X. Liu, H. Hesselbom, L. Akesso, M. Fahlman, X. Crispin, I. Engquist, M. Berggren and G. Gustafsson, Proc. Natl. Acad. Sci. U.S.A., 2014, 111, 11943-11948.

203 A. Turner, Chem. Soc. Rev., 2013, 42, 3184-3196.

204 S. Lee, A. Aranyosi, M. D. Wong, J. H. Hong, J. Lowe, C. Chan, D. Garlock, S. Shaw, P. D. Beattie, Z. Kratochvil, N. Kubasti, K. Seagers, R. Ghaffari and C. D. Swanson, Biosens. Bioelectron., 2016, 78, 290-299.

205 T. Ahmadraji, L. Gonzalez-Macia, T. Ritvonen, A. Willert, S. Ylimaula, D. Donaghy, S. Tuurala, M. Suhonen, D. Smart, A. Morrin, V. Efremov, R. R. Baumann, M. Raja, A. Kemppainen and A. J. Killard, Anal. Chem., 2017, 89, 7447-7454.

206 R. Vyas, V. Lakafosis, A. Rida, N. Chaisilwattana, S. Travis, J. Pan and M. Tentzeris, IEEE Trans. Microwave Theory Tech., 2009, 57, 1370-1382.

207 L. Yang, R. Zhang, D. Staiculescu, C. Wong and M. Tentzeris, IEEE Antennas Wirel. Propag. Lett., 2009, 8, 653-656.

208 D. D. Liana, B. Raguse, J. J. Gooding and E. Chow, Adv. Mater. Technol., 2016, 1, 1600143. 
209 D. D. Liana, B. Raguse, J. J. Gooding and E. Chow, Anal. Methods, 2017, 9, 66-75.

210 DFA: Diagnostics for All, https://dfa.org/, 2018.

211 INSiGHT: Institute for Nutritional Sciences, Global Health, and Technology, 2018, https://insight.cornell.edu.
212 A. A. Kumar, J. W. Hennek, B. S. Smith, S. Kumar, P. Beattie, S. Jain, J. P. Rolland, T. P. Stossel, C. Chunda-Liyoka and G. M. Whitesides, Angew. Chem., Int. Ed., 2015, 54, 58365853. 